\title{
THE $l_{1}$-ALGEBRA OF A COMMUTATIVE SEMIGROUP
}

\author{
BY \\ EDWIN HEWITT AND HERBERT S. ZUCKERMAN(1)
}

\section{Introduction.}

1.1 The present paper may be regarded as a chapter in the theory of convolution algebras inaugurated in [3]. The algebras dealt with here are in general infinite dimensional commutative Banach algebras, so that the apparatus of Gel'fand's theory [2], topological considerations, and a number of analytic devices all play a rôle. It will be seen, however, that the functionals making up the algebras under study have obvious representations as integrals with respect to countably additive, totally finite measures that vanish except on countable sets. Thus the refinements of measure and integration theory are avoided, nothing more recondite than infinite series being needed for all of the integral computations employed. The great majority of our results deal only with the commutative case: in view of the genuine difficulties connected with harmonic analysis even on noncommutative groups and the obvious fact that semigroups are less tractable than groups, no apology is perhaps required for this.

The present paper may be described as an introduction to harmonic analysis on discrete commutative semigroups. It will no doubt be noted that we state no theorems concerning Tauberian theorems, analogues of the Pontryagin duality theorem, or the Silov boundary. We hope to deal with these topics in a subsequent communication.

1.2. Throughout this paper, we use the terminology, notions, and results of [3]. A few points, however, require re-statement, and we make a few new

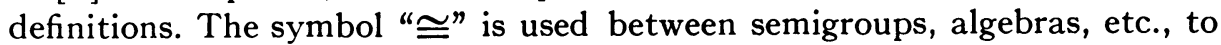
denote the existence of a 1-to- 1 correspondence $\pi$ preserving all operations of both systems; and if a product, say, is defined only for some pairs, then the equality $\pi(x y)=\pi(x) \pi(y)$ is to hold whenever either side is defined. A homomorphism of a semigroup $G$ onto a semigroup $H$ is a single-valued mapping $\mu$ of $G$ onto $H$ such that $\mu(x y)=\mu(x) \mu(y)$ for all $x, y \in G$.

1.3. A multiplicative function on a semigroup $G$ is any complex function $f$ on $G$ satisfying the functional equation $f(x y)=f(x) f(y)$ for all $x, y \in G$. A multiplicative function is called a semicharacter (see $[3,3.1]$ ) if it is different from 0 at some point and is bounded. It is easy to see that if $\chi$ is a semicharacter on $G$, then $|\chi(x)| \leqq 1$ for all $x \in G$, and that a semicharacter of a group

Received by the editors August 5, 1955.

(1) The authors wish to acknowledge financial assistance by the National Science Foundation during the preparation of this paper. 
is a character. A group, however, may easily admit unbounded multiplicative functions.

1.4. Let $X$ be any nonvoid set, $x$ any point of $X$, and $\mathfrak{F}$ any linear space of complex-valued functions on $X$. The linear functional on $\mathfrak{F}$ whose value at $f \in \mathfrak{F}$ is $f(x)$ will be denoted by $\lambda_{x}$ : thus $\lambda_{x}(f)=f(x)$. We shall be largely concerned in this paper with functionals of the form $\sum_{x \in X} \alpha(x) \lambda_{x}$, where $\alpha$ is a complex-valued function on $X$ such that $\sum_{x \in X}|\alpha(x)|$ is finite. By this last we mean that the set $\{x ; x \in X, \alpha(x) \neq 0\}$ is countable, being written say as $\left\{x_{1}, x_{2}, x_{3}, \cdots, x_{n}, \cdots\right\}$, and that $\sum_{n=1}^{\infty}\left|\alpha\left(x_{n}\right)\right|$ is finite. The number $\sum_{n=1}^{\infty}\left|\alpha\left(x_{n}\right)\right|$ is obviously independent of the order in which $\left\{x_{1}, x_{2}, x_{3}, \cdots\right.$, $\left.x_{n}, \cdots\right\}$ is taken. We use the convention that an expression of the form $\sum_{n=1}^{\infty} u_{n}$ may denote either a finite or an infinite series and that a sum over the void set is 0 .

2. Definition and elementary properties of $l_{1}(G)$.

2.1. Definition. Let $G$ be an arbitrary semigroup. Let $\mathfrak{B}(G)$ denote the linear space of all bounded complex-valued functions on $G$. Let $l_{1}(G)$ denote the set of all functionals $A$ on $\mathfrak{B}(G)$ having the form

2.1 .1

$$
A=\sum_{x \in G} \alpha(x) \lambda_{x},
$$

where $\alpha$ is a complex-valued function on $G$ for which $\sum_{x \in G}|\alpha(x)|$ is finite.

2.2. TheOREM. The set $l_{1}(G)$ of 2.1 is a linear space of bounded linear functionals on $\mathfrak{B}(G)$. There is a norm-preserving linear isomorphism between $l_{1}(G)$ and the Banach space of all complex-valued functions $\alpha$ on $G$ that vanish except on countable subsets of $G$ and for which

$$
\sum_{x \in G}|\alpha(x)|=\|\alpha\|
$$

is finite.

Proof. For $f \in \mathfrak{B}(G)$, with $\|f\|=\sup _{x \in G}|f(x)|$, and $A \in l_{1}(G)$, we have $|A(f)|=\left|\sum_{x \in G} \alpha(x) f(x)\right| \leqq\|f\| \sum_{x \in G}|\alpha(x)|$. Hence $A$ is a bounded linear functional and $\|A\| \leqq \sum_{x \in G}|\alpha(x)|$. Next, let $f \in \mathfrak{B}(G)$ be defined by the relations $f(x)=0$ if $\alpha(x)=0$ and $f(x)=\bar{\alpha}(x)|\alpha(x)|^{-1}$ if $\alpha(x) \neq 0$, where $\bar{\alpha}(x)$ is the complex conjugate of $\alpha(x)$. Then if $f \neq 0$, we have $\|f\|=1$. Since $A(f)$ $=\sum_{x \in G}|\alpha(x)|$, it follows that

$$
\|A\|=\sum_{x \in G}|a(x)| .
$$

The rest of the present theorem is obvious.

2.3. Theorem. The space $l_{1}(G)$ is a convolution algebra. For $A$ $=\sum_{u \in G} \alpha(u) \lambda_{u}$ and $B=\sum_{v \in G} \beta(v) \lambda_{v}$ in $l_{1}(G), A * B$ is the functional $\sum_{x \in G} \alpha * \beta(x) \lambda_{x}$, where 


$$
\alpha * \beta(x)=\sum_{u, v, u v=x} \alpha(u) \beta(v)
$$

for all $x \in G$.

Proof. Let $f$ be any function in $\mathfrak{B}(G)$. It is clear that ${ }_{x} f \in \mathfrak{B}(G)$ for all $x \in G:|f(x y)| \leqq \sup _{u \in G}|f(u)|$ for all $x, y \in G$. This is condition 1.3.1 of [3]. For $B \in l_{1}(G)$ as above, we have

$$
\left|B\left({ }_{u} f\right)\right|=\left|\sum_{v \in G} \beta(v) \lambda_{v}(u f)\right|=\left|\sum_{r \in G} \beta(v) f(u v)\right| \leqq\|B\|\|f\| .
$$

Hence $B\left({ }_{x} f\right)$ is a bounded function of $x$; this establishes condition 1.3.2 of [3]. To verify condition 1.3 .3 of [3], we argue as follows. For every $u \in G$, the number $\sum_{v \in G} \beta(v) f(u v)$ is the value at $u$ of the function $B\left({ }_{u} f\right)$. Hence, to compute $A * B(f)$, we write

$$
A * B(f)=\sum_{u \in G} \alpha(u) \lambda_{u}\left(B\left({ }_{u} f\right)\right)=\sum_{u \in G} \alpha(u)\left[\sum_{v \in G} \beta(v) f(u v)\right] .
$$

Since

$$
\sum_{u \in G}|\alpha(u)|\left[\sum_{v \in G}|\beta(v)| \cdot|f(u v)|\right] \leqq\|A\| \cdot\|B\| \cdot\|f\|<\infty,
$$

we may rearrange the last expression in 2.3.2 in any fashion without altering its value. Let $\left\{x_{1}, x_{2}, x_{3}, \cdots, x_{n}, \cdots\right\}$ be the (finite or countably infinite) set of all distinct products $u v$ for which $\alpha(u) \neq 0$ and $\beta(v) \neq 0$. Then we have

$$
\sum_{u \in G} \alpha(u)\left[\sum_{v \in G} \beta(v) f(u v)\right]=\sum_{n=1}^{\infty}\left[\sum_{u, v, u v=x_{n}} \alpha(u) \beta(v)\right] f\left(x_{n}\right) .
$$

Finally, we may write

$$
A * B(f)=\sum_{x \in G}\left[\sum_{u, e, u v=x} \alpha(u) \beta(v)\right] f(x)=\sum_{x \in G} \alpha * \beta(x) f(x),
$$

with the usual convention that a void sum is 0 . It remains to show that the functional $\sum_{x \in G} \alpha * \beta(x) \lambda_{x}$ is in $l_{1}(G)$, i.e., that

$$
\sum_{x \in G}|\alpha * \beta(x)|<\infty .
$$

To this end, consider the triple sum

$$
\sum_{u \in G}|\alpha(u)|\left\{\sum_{x \in G}\left[\sum_{v, u v=x}|\beta(v)|\right]\right\} .
$$

For every fixed $u$, the sum $\{\cdots\}$ is less than or equal to $\sum_{v \in G}|\beta(v)|$, since each $v \in G$ can appear at most once in a summand $[\cdots]$. It follows that 2.3.6 
is less than or equal to $\|A\| \cdot\|B\|$, a finite number, and we may therefore rearrange 2.3.6 in any fashion without altering its value. It follows that

$$
\begin{aligned}
\sum_{x \in G}|\alpha * \beta(x)| & =\sum_{x \in G}\left|\sum_{u, v, u v=x} \alpha(u) \beta(v)\right| \\
& \leqq \sum_{x \in G}\left\{\sum_{u, v, u v=x}|\alpha(u)||\beta(v)|\right\} \\
& =\sum_{u \in G}|\alpha(u)|\left\{\sum_{x \in G}\left[\sum_{v, u v=x}|\beta(v)|\right] \leqq\|A\| \cdot\|B\| \leqq \infty .\right.
\end{aligned}
$$

This inequality is just 2.3.5, and the proof is complete.

2.4. Theorem. With the norm $\|A\|, l_{1}(G)$ is a Banach algebra.

Proof. This follows from 2.3.7 and the fact that $\|A * B\|=\sum_{x \in G}|\alpha * \beta(x)|$.

2.5. Note. According to Gel'fand's original definition $[2$, p. 3$]$, if a Banach algebra has a unit, then the norm of the unit is 1 . This need not be the case with the definition just given for the norm in $l_{1}(G)$, as 11.1.6 shows. Of course $l_{1}(G)$ can be renormed so that the unit, if one exists, has norm 1 .

2.6. Theоrem. Let $\left\{u_{1}, \cdots, u_{p}\right\}$ and $\left\{v_{1}, \cdots, v_{q}\right\}$ be finite subsets of $G$ and $\left\{\alpha_{1}, \cdots, \alpha_{p}\right\},\left\{\beta_{1}, \cdots, \beta_{q}\right\}$ sequences of complex numbers. Then

2.6 .1

$$
\left(\sum_{j=1}^{p} \alpha_{j} \lambda_{u_{j}}\right) *\left(\sum_{k=1}^{q} \beta_{k} \lambda_{v_{k}}\right)=\sum_{j=1}^{p} \sum_{k=1}^{q} \alpha_{j} \beta_{k} \lambda_{u_{j} k_{k}} .
$$

Proof. It is obvious that $\sum_{j=1}^{p} \alpha_{j} \lambda_{u_{j}}$ and $\sum_{k=1}^{q} \beta_{k} \lambda_{v_{k}}$ are elements of $l_{1}(G)$. Consider first the product $\lambda_{u} * \lambda_{v}$, where $u$ and $v$ are arbitrary elements of $G$. Applying 2.3.1, we see that the only nonzero number $\alpha * \beta(x)$ is $\alpha * \beta(u v)=1$, and hence $\lambda_{u} * \lambda_{v}=\lambda_{u v}$. Formula 2.6.1 now follows by the distributive law.

We next identify all multiplicative linear functionals on $l_{1}(G)$. This is of course equivalent to finding all maximal regular 2 -sided ideals $\mathfrak{N}$ such that $l_{1}(G)-\mathscr{T C}$ is commutative.

2.7. Theorem. Let $G$ be an arbitrary semigroup, and let $\tau$ be a multiplicative linear functional on $l_{1}(G)$ different from 0 . Then $\tau$ is a bounded linear functional, and there exists a semicharacter $\chi$ of $G$ such that, for all $A=\sum_{x \in G} \alpha(x) \lambda_{x} \in l_{1}(G)$, we have

2.7 .1

$$
\tau(A)=\sum_{x \in G} \alpha(x) \chi(x) .
$$

Conversely, every semicharacter $\chi$ of $G$ defines a bounded multiplicative linear functional by 2.7.1, and 2 distinct semicharacters define 2 distinct multiplicative linear functionals. The norm $\|\tau\|$ of $\tau$ as a linear functional is equal to $\sup _{x \in G}|\chi(x)|$. 
Proof. Let $\tau$ be a nonzero multiplicative linear functional on $l_{1}(G)$. It is well known that $\tau$ is continuous. (See for example [4, p. 481, Theorem 22.14.1].) Obviously the function $\chi$ defined by $\chi(x)=\tau\left(\lambda_{x}\right)$ is a semicharacter of $G$. Since $\tau$ is continuous, 2.7.1 must hold for all $A \in l_{1}(G)$. The rest of the present theorem is similarly easy to establish.

The preceding theorem enables us to identify the radical of $l_{1}(G)$ for commutative $G$.

2.8. TheOREM. Let $G$ be a commutative semigroup, and let $\odot$ be the Jacobson radical of $l_{1}(G)$ [4, p. 476, Definition 22.13.2]. Then $\odot$ consists of all $A=\sum_{x \in G} \alpha(x) \lambda_{x} \in l_{1}(G)$ such that $\sum_{x \in G} \alpha(x) \chi(x)=0$ for all $\chi \in \widehat{G}[3,3.7]$. In particular, $l_{1}(G)$ is semisimple if and only if for every complex-valued function $\alpha \neq 0$ on $G$ such that $\sum_{x \in G}|\alpha(x)|<\infty$, there exists some $\chi \in \widehat{G}$ for which $\sum_{x \in G} \alpha(x) \chi(x) \neq 0$.

Proof. $l_{1}(G)$ is not a radical algebra, since it contains the proper regular maximal ideal $\left\{A ; A \in l_{1}(G), \sum_{x \in G} \alpha(x)=0\right\}$. (This is the kernel of the multiplicative linear functional $\sum_{x \in G} \alpha(x) \lambda_{x} \rightarrow \sum_{x \in G} \alpha(x)$.) Hence by [4, p. 485, Theorem 22.15.1 ], $\odot$ is the intersection of all proper regular maximal ideals $\mathfrak{T}$ of $l_{1}(G)$. However, $l_{1}(G)-\mathfrak{T}$ is the complex field, as is well known (see for example $[5$, p. $69,23 \mathrm{~A}]$ ) and $\mathfrak{T}$ is thus the kernel $\mathfrak{M}_{\tau}$ of a multiplicative linear functional $\tau$. Combining this observation with 2.7 , we obtain the present theorem.

2.9. Remark. Theorem 2.8 shows that the maximal ideal space of $l_{1}(G)$ for a commutative semigroup $G$ can be identified with $\widehat{G}$, just as in the case where $G$ is a group. We postpone to $\$ 8$ a discussion of the topology that can be imposed from this point of view on $\widehat{G}$.

2.10. REMARK. It is natural to look for function spaces other than $\mathfrak{B}(G)$ that can serve as the space $\mathfrak{F}$ of $[3,1.3]$, in order to realize $l_{1}(G)$ as a convolution algebra. Obvious choices are the space $\mathfrak{E}_{\infty \infty \infty}(G)$ consisting of all complexvalued functions on $G$ that vanish outside of finite subsets of $G$ and the space $\mathfrak{C}_{\infty}(G)$ consisting of all complex-valued functions $f$ on $G$ such that for every real number $\delta>0$, there exists a finite subset $A$ of $G$ such that $|f(x)|<\delta$ for all $x \notin A$. If there exist $a, b \in G$ such that the equation $a y=b$ has an infinite number of solutions $y \in G$, then if $f(b)=1$, say, we have $a f(y)=1$ for an infinite number of elements $y$. It is clear that $[3,1.3 .1]$, is violated in this case. On the other hand, if the equation $a y=b$ has no solution or only a finite number of solutions for all $a, b \in G$, then the function spaces $\mathfrak{S}_{\infty \infty \infty}(G)$ and $\mathfrak{E}_{\infty}(G)$ both satisfy $[3,1.3 .1]$, and may be used as the space $\mathfrak{F}$ for $l_{1}(G)$. For examples of semigroups in which the equation $a y=b$ admits an infinite number of solutions for some $a$ and $b$, see 11.1.3.

3. A criterion for semisimplicity of $l_{1}(G)$.

3.1. In this section, we establish a useful necessary and sufficient condition that $l_{1}(G)$ be semisimple, if $G$ is commutative. The main theorem of this sec- 
tion, 3.4, is appropriately stated for spaces of functions on sets without any semigroup structure. Throughout this section, then, $X$ will denote an arbitrary nonvoid set, finite or infinite (although our theorem is trivial for $X$ finite). The expression $l_{1}(X)$ will denote the space of all complex-valued functions $\alpha$ on $X$ such that $\sum_{x \in X}|\alpha(x)|$ is finite. The symbol $\subseteq$ will denote a set of complex-valued functions on $X$ satisfying the following conditions:

3.1.1 for all $\phi \in \subseteq$ and $x \in X,|\phi(x)| \leqq 1$;

3.1.2 the function 1 is in $\mathfrak{S}$;

3.1.3 if $\phi_{1}, \phi_{2}$ are in $\mathfrak{S}$, then $\phi_{1} \phi_{2} \in \mathfrak{S}$ or $\phi_{1} \phi_{2}=0$;

3.1.4 if $\phi \in \mathfrak{S}$, then $\phi \in \mathfrak{S}$.

Let $\mathfrak{T}$ denote the linear space of all finite complex linear combinations of functions in $\mathfrak{S}$. It is obvious that $\mathfrak{T}$ is an algebra of bounded complex-valued functions having the property that $\psi \in \mathfrak{T}$ implies $|\psi|^{2} \in \mathfrak{T}$.

3.2. Definition. A family of complex-valued functions defined on $X$ is said to be a separating family on $X$ or to separate points of $X$ if, for every pair of distinct points $x, y \in X$, there is a function $f$ in the family under consideration such that $f(x) \neq f(y)$.

3.3. Theorem. Let $X, l_{1}(X)$, and $\mathfrak{S}$ be as in 3.1. Suppose that for every $\alpha \in l_{1}(X), \alpha \neq 0$, there exists an element $\phi$ of $\subseteq$ such that $\sum_{x \in G} \alpha(x) \phi(x) \neq 0$. Then $\mathfrak{S}$ is a separating family on $X$.

Proof. If there exist distinct points $a, b \in X$ such that $\phi(a)=\phi(b)$ for all $\phi \in \mathfrak{S}$, let $\alpha(a)=1, \alpha(b)=-1$, and $\alpha(x)=0$ for all other $x \in X$. Plainly $\alpha \in l_{1}(X)$ and $\sum_{x \in G} \alpha(x) \phi(x)=\phi(a)-\phi(b)=0$ for all $\phi \in \mathfrak{S}$.

The converse of the foregoing obvious assertion is true, but its proof lies somewhat deeper. We now state and prove it.

3.4. TheOREM. Let $X, l_{1}(X)$, and $\subseteq$ be as in 3.1, and suppose that $\subseteq$ is a separating family on $X$. Then for every $\alpha \in l_{1}(X)$ that is different from 0 , there exists a function $\phi \in \mathbb{S}$ such that $\sum_{x \in x} \alpha(x) \phi(x) \neq 0$.

Proof. Assume that the theorem is false: then for some $\alpha \neq 0, \alpha \in l_{1}(X)$, we have $\sum_{x \in X} \alpha(x) \phi(x)=0$ for all $\phi \in \mathbb{S}$ and hence $\sum_{x \in X} \alpha(x) \phi(x)=0$ for all $\phi \in \mathfrak{T}$. We may order the set $\{x ; x \in X, \alpha(x) \neq 0\}$ in the form $\left\{x_{1}, x_{2}\right.$, $\left.x_{3}, \cdots, x_{n}, \cdots\right\}$, and for convenience we write $\alpha\left(x_{j}\right)=\alpha_{j}(j=1,2,3, \cdots)$. Now let $r$ be an arbitrary positive integer. For every integer $j, 2 \leqq j \leqq r$, there exists $\phi_{j} \in \mathfrak{S}$ such that $\phi_{j}\left(x_{1}\right) \neq \phi_{j}\left(x_{j}\right)$, since $\subseteq$ separates points in $X$. Let $\psi$ be the function such that

$$
\psi(x)=\prod_{j=2}^{r} \frac{\phi_{j}(x)-\phi_{j}\left(x_{j}\right)}{\phi_{j}\left(x_{1}\right)-\phi_{j}\left(x_{j}\right)}
$$

for all $x \in X$. It is clear from 3.1.2 and 3.1.3 that $\psi \in \mathfrak{T}$. It is also easy to see that $\psi\left(x_{1}\right)=1$ and $\psi\left(x_{j}\right)=0(j=2,3, \cdots, r)$. For every real number $t$ and 
every non-negative integer $n$, the function

$$
\frac{\left(i t|\psi|^{2}\right)^{n}}{n !}
$$

is an element of $\mathfrak{T}$. It follows from our assumption, then, that

$$
\sum_{j=1}^{\infty} \alpha_{j} \frac{\left[i t\left|\psi\left(x_{j}\right)\right|^{2}\right]^{n}}{n !}=0 .
$$

Summing 3.4.1 with respect to $n$, we obtain

$$
\sum_{n=0}^{\infty}\left\{\sum_{j=1}^{\infty} \alpha_{j} \frac{\left[i t\left|\psi\left(x_{j}\right)\right|^{2}\right]^{n}}{n !}\right\}=0 .
$$

Since the series $\sum_{j=1}^{\infty}\left|\alpha_{j}\right|$ converges and since $\psi$ is a bounded function, the double series 3.4 .2 converges absolutely, and we may rearrange the order of summation at will without altering its value. Thus

$$
\sum_{j=1}^{\infty} \alpha_{j}\left\{\sum_{n=0}^{\infty} \frac{\left[i t\left|\psi\left(x_{j}\right)\right|^{2}\right]^{n}}{n !}\right\}=0 ;
$$

equivalently,

$$
\sum_{j=1}^{\infty} \alpha_{j} \exp \left(i t\left|\psi\left(x_{j}\right)\right|^{2}\right)=0 .
$$

Consider the left side of 3.4.4 as a function $g(t)(-\infty<t<+\infty)$. This function $g$ is a Bohr almost periodic function (with absolutely convergent Fourier series). By 3.4.4, we have $g=0$; by the uniqueness theorem for almost periodic functions (see for example [6, p. 117]), all Fourier coefficients of $g$ must vanish. (In this simple case, one can also take the Bohr mean value of $g(t) e^{i s t}$ for real s.) The Fourier coefficient of $g$ for the exponent 1 is a sum $\sum_{j} \alpha_{j}$, the sum being taken over all $j$ such that $\left|\psi\left(x_{j}\right)\right| .=1$. For $1 \leqq j \leqq r$, the only entry in this sum is $\alpha_{1}$. It follows that $\left|\alpha_{1}\right| \leqq \sum_{j=r+1}^{\infty}\left|\alpha_{j}\right|$. Since $r$ is arbitrary, we infer that $\alpha_{1}=0$. This contradicts the definition of $x_{1}$, and hence we see that $\alpha=0$. This completes the proof.

Theorems 3.3 and 3.4 yield the following criterion for semisimplicity of $l_{1}(G), G$ commutative.

3.5. Theorem. Let $G$ be a commutative semigroup. Then $l_{1}(G)$ is semisimple if and only if the set $\widehat{G}$ of semicharacters is a separating family on $G$.

Proof. An element $A=\sum_{x \in G} \alpha(x) \lambda_{x}$ of $l_{1}(G)$ is in the radical of $l_{1}(G)$ if and only if $\sum_{x \in G} \alpha(x) \chi(x)=0$ for all $\chi \in \widehat{G}$, by 2.8 . The set of functions $\widehat{G}$ obviously satisfies conditions $3.1 .1-3.1 .4$; thus 3.3 and 3.4 yield the present theorem. 
4. Certain algebraic properties of commutative semigroups. Theorem 2.8 shows an intimate connection between the radical of $l_{1}(G)$ and the semicharacters of $G$. In this section, we establish various algebraic properties of $G$ which will be used in $\$ 5$ to construct semicharacters of $G$. These in turn, with 2.8 and 3.5 , will be used to identify the radical of $l_{1}(G)$. We first break up the semigroup $G$ into subsets $T_{x}$ which, as we shall show later, have the property that $y \in T_{x}$ and if only if $\chi(x)=\chi(y)$ for all $\chi \in \widehat{G}$.

4.1. Definition. Let $G$ be a commutative semigroup. For $x \in G$, let $T_{x}$ be the set $T_{x}=\left\{y ; y \in G, x y^{n}=y^{n+1}, y x^{n}=x^{n+1}\right.$, for some positive integer $\left.n\right\}$.

It is obvious that $x \in T_{x}$ for all $x \in G$ and that $y \in T_{x}$ implies $x \in T_{y}$. Furthermore, if $z \in T_{y}$ and $y \in T_{x}$, then we have $y z^{n}=z^{n+1}, z y^{n}=y^{n+1}, y x^{m}$ $=x^{m+1}$, and $x y^{m}=y^{m+1}$ for some positive integers $m$ and $n$. From these equalities, we find $x z^{(n+1)(m+1)-1}=x z^{(n+1) m} z^{n}=x y^{m} z^{n m} z^{n}=y^{m+1} z^{n(m+1)}=z^{(n+1)(m+1)}$ and $z x^{(n+1)(m+1)-1}=z x^{(m+1) n} x^{m}=z y^{n} x^{n m} x^{m}=y^{n+1} x^{m(n+1)}=x^{(n+1)(m+1)}$. It follows that $z \in T_{x}$. These results imply the following theorem.

4.2. Theorem. The commutative semigroup $G$ is the union of the sets $T_{x}$. Every two of these sets are either identical or disjoint. Also, $x \in T_{x}$ for all $x \in G$.

4.3. Theorem. For all $x, y, z$, and $w$ in $G$. if $y \in T_{x}$ and $z \in T_{w}$, then $y z \in T_{x w}$.

Proof. If $y \in T_{x}$, then $x y^{n}=y^{n+1}$ and $y x^{n}=x^{n+1}$. Hence $(z x)(z y)^{n}=(z y)^{n+1}$ and $(z y)(z x)^{n}=(z x)^{n+1}$. Therefore $y z \in T_{x z}$. Hence, if $y \in T_{x}$ and $z \in T_{w}$, then $y z \in T_{x z}$ and $x z \in T_{x w}$; from this, we infer that $y z \in T_{x w}$.

4.4. Definition. Let $G^{\prime}$ be the set whose elements are all of the distinct sets $T_{x}, G^{\prime}=\left\{T_{x}\right\}$, with the operation $T_{x} T_{y}=T_{x y}$. It is clear that $G^{\prime}$ is a commutative semigroup and that the mapping $x \rightarrow T_{x}$ is a homomorphism of $G$ onto $G^{\prime}$.

4.5. Theorem. Let $T_{x}, T_{y}$ be elements of $G^{\prime}$. Then, if $T_{x} T_{y}=T_{x}^{2}=T_{y}^{2}$, it follows that $T_{x}=T_{y}$.

Proof. From 4.4, we have $T_{x y}=T_{x^{2}}=T_{y^{2}}$ and hence $x^{2} \in T_{x y}$ and $\dot{y}^{2} \in T_{x y}$. From the first of these relations, we have $(x y) x^{2 n}=x^{2(n+1)}$ and, from the second, $(x y) y^{2 m}=y^{2(m+1)}$. Therefore we have $y x^{2 n+1}=x^{2 n+2}$ and $x y^{2 m+1}=y^{2 m+2}$, $y x^{2(n+m)+1}=x^{2(n+m)+2}$ and $x y^{2(n+m)+1}=y^{2(n+m)+2}$, and therefore $x \in T_{y}$. This implies that $T_{x}=T_{y}$.

4.6. The property proved in 4.5 will be shown to imply that $l_{1}\left(G^{\prime}\right)$ is semisimple. With this in mind, we note the following two theorems.

4.7. Theorem. Let $G$ be a commutative semigroup with the property that $x y=x^{2}=y^{2}$ implies $x=y$, for all $x, y \in G$. Then, for all $x, y, z$ in $G$, if $x^{n} y=x^{n} z$ for some positive integer $n$, it follows that $x y=x z$.

Proof. Suppose first that $n$ is odd. In this case, let $m=(n+1) / 2$. We have 
$\left(x^{m} y\right)^{2}=x^{n+1} y^{2}=x x^{n} y y=x x^{n} z y=x x^{n} z^{2}$. Suppose next that $n$ is even. In this case, let $m=n / 2$. We then have $\left(x^{m} y\right)^{2}=x^{n} y^{2}=x^{n} z y=x^{n} z^{2}$. In both cases, we have $\left(x^{m} y\right)^{2}=\left(x^{m} y\right)\left(x^{m} z\right)=\left(x^{m} z\right)^{2}$ and hence $x^{m} y=x^{m} z$. Since $m$ is a positive integer such that $m<n$ for $n>1$, we can repeat this argument until we obtain $x y=x z$.

4.8. Theorem. Let $G$ be a commutative semigroup. Then it has the property that $x y=x^{2}=y^{2}$ implies $x=y$, if and only if all of its subsets $T_{x}$ consist of precisely one element.

Proof. This follows easily from 4.1 and 4.7. We omit the details.

4.9. Now we break up the semigroup $G^{\prime}$ into a certain family of pairwise disjoint subsets. These subsets will be subsemigroups in which the cancellation law holds. It is convenient to change the notation and to consider $G^{\prime}$ as a commutative semigroup with elements $x, y, \ldots$ and having the property that $x y=x^{2}=y^{2}$ implies $x=y$.

4.10. Definition. For all $x \in G^{\prime}$, let $H_{x}$ be the set $H_{x}=\left\{y ; y \in G^{\prime}, y^{n}=u x\right.$, $x^{n}=v y$ for some positive integer $n$ and some $u \in G^{\prime}$ and $\left.v \in G^{\prime}\right\}$.

The following properties of the sets $H_{x}$ are easy to verify: $x \in H_{x} ; x^{2} \in H_{x}$; $y \in H_{x}$ implies $x \in H_{y} ; z \in H_{y}$ and $y \in H_{x}$ imply $z \in H_{x} ; y \in H_{x}$ and $z \in H_{w}$ imply $y z \in H_{x w}$. These relations imply the next two theorems.

4.11. Theorem. The semigroup $G^{\prime}$ is the union of the subsets $H_{x}$. Every two of these sets are either identical or disjoint, and $x \in H_{x}$ for all $x \in G^{\prime}$.

4.12. Definition. Let $G^{\prime \prime}$ be the set whose elements are all of the distinct sets $H_{x}, G^{\prime \prime}=\left\{H_{x}\right\}$, with the operation $H_{x} H_{y}=H_{x y}$.

4.13. Theorem. $G^{\prime \prime}$ is an idempotent commutative semigroup. The mapping $x \rightarrow H_{x}$ is a homomorphism of $G^{\prime}$ onto $G^{\prime \prime}$. Furthermore, every set $H_{x}$ is a subsemigroup of $G^{\prime}$.

4.14. Theorem. If $y \in H_{x}$, then there exist elements $u^{\prime}$ and $v^{\prime}$ of $H_{x}$ and a positive integer $n^{\prime}$ such that $y^{n^{\prime}}=u^{\prime} x$ and $x^{n^{\prime}}=v^{\prime} y$.

Proof. We have $y^{n}=u x$ and $x^{n}=v y$ for some $u, v \in G^{\prime}$, and hence $y^{n+1}$ $=(u y) x$ and $x^{n+1}=(v x) y$. Now $H_{u y}=H_{u} H_{y}=H_{u} H_{x}$ and hence $u y \in H_{u y}$ $=H_{u} H_{x}=H_{u} H_{x}^{2}=H_{u y} H_{x}=H_{u y x}=H_{y}^{n+1}=H_{y}=H_{x}$. Similarly we find $v x \in H_{x}$. This gives us the present theorem with $u^{\prime}=u y, v^{\prime}=v x$, and $n^{\prime}=n+1$.

4.15. THEOREM. If $y \in H_{x}, z \in H_{x}$, and $x y=x z$, then $y=z$.

Proof. By 4.10 , we have $y^{n}=u x, z^{m}=w x$, and therefore $y^{n+1}=u x y=u x z$ $=y^{n} z, z^{m+1}=w x z=w x y=z^{m} y$. From 4.9 and 4.7 , we find $y^{2}=y z, z^{2}=y z$ and then $y=z$.

4.15.1. CoROllaRy. The cancellation law holds in every subsemigroup $H_{x}$. 
4.16. THEOREM. If $H_{x}$ contains an idempotent element e, then $H_{x}=H_{0}$ is a group.

Proof. If $y \in H_{e}$, then $e^{2} y=e y$ and $e y=y$ by 4.15. Also, by $4.14, e^{n}=v y$ for some $v \in H_{e}$, and therefore $v y=e$. Since $H_{e}$ is a semigroup (4.13), it is a group.

4.17. If all of the subsemigroups $H_{x}$ were groups, our problem would be somewhat simpler. Elementary examples (see 11.6) show that this is not always the case. For our study of semigroups with semisimple $l_{1}$-algebras and for the identification of the radical in other $l_{1}$-algebras, we find it useful to imbed $G^{\prime}$ in a semigroup $G^{0}$ that is the union of groups. The first step in this imbedding process is to consider all ordered pairs $(x, y)$ with $x \in G^{\prime}$, $y \in H_{x}$, and the operation $(x, y)(z, w)=(x z, y w)$.

4.18. ThÉorem. For pairs $(x, y)$ and $(w, z)$ as in 4.17 , let $(x, y) \sim(w, z)$ mean that $w \in H_{x}$ and $x z=y w$. The relation $\sim$ is then an equivalence relation.

Proof. It is obvious that the relation is reflexive and symmetric. We show that it is transitive. If $(x, y) \sim\left(x^{\prime}, y^{\prime}\right)$ and $\left(x^{\prime}, y^{\prime}\right) \sim\left(x^{\prime \prime}, y^{\prime \prime}\right)$, then all 6 elements $x, y, \cdots, y^{\prime \prime}$ are in $H_{x}=H_{y^{\prime}}$. Also we have $y^{\prime}\left(x y^{\prime \prime}\right)=\left(x y^{\prime}\right) y^{\prime \prime}=\left(y x^{\prime}\right) y^{\prime \prime}$ $=y\left(x^{\prime} y^{\prime \prime}\right)=y\left(y^{\prime} x^{\prime \prime}\right)=y^{\prime}\left(x^{\prime \prime} y\right)$. By 4.15, we then have $x y^{\prime \prime}=x^{\prime \prime} y$.

4.18.1. THEOREM. If $(x, y) \sim\left(x^{\prime}, y^{\prime}\right)$ and $(z, w) \sim\left(z^{\prime}, w^{\prime}\right)$, then $(x, y)(z, w)$ $\sim\left(x^{\prime}, y^{\prime}\right)\left(z^{\prime}, w^{\prime}\right)$.

Proof. We have $(x, y)(z, w)=(x z, y w),\left(x^{\prime}, y^{\prime}\right)\left(z^{\prime}, w^{\prime}\right)=\left(x^{\prime} z^{\prime}, y^{\prime} w^{\prime}\right)$, and $(x z)\left(y^{\prime} w^{\prime}\right)=\left(x y^{\prime}\right)\left(z w w^{\prime}\right)=\left(y x^{\prime}\right)\left(w z^{\prime}\right)=(y w)\left(x^{\prime} z^{\prime}\right)$. Furthermore, we have $x^{\prime} z^{\prime}$ $\in H_{x^{\prime}} H_{z^{\prime}}=H_{x} H_{z}=H_{x z}$.

4.19. Definition. For all $x \in G^{\prime}$ and $y \in H_{x}$, let $[x, y]=\{(z, w) ;(z, w)$ $\sim(x, y)\}$. Let $G^{0}$ be the set whose elements are all of the distinct sets $[x, y]$, with the operation $[x, y][z, w]=[x z, y w]$. It is clear that $G^{0}$ is a commutative semigroup.

4.20. Definition. For all $x \in G^{\prime}$, let $H_{x}^{0}=\left\{[y, z] ;[y, z] \in G^{0}, y \in H_{x}\right\}$.

4.21. Theorem. The semigroup $G^{0}$ is the union of the sets $H_{x}^{0}$. Every two sets $H_{x}^{0}$ are identical or disjoint, and each $H_{x}^{0}$ is a group. $H_{x}^{0}$

Proof. If $[x, y] \in G^{0}$, then $[x, y] \in H_{x}^{0}$. Therefore $G^{0}$ is the union of the sets

If $[x, y] \in H_{z}^{0} \cap H_{w}^{0}$, then $x \in H_{z}$ and $x \in H_{w}$. Hence $H_{x}=H_{z}=H_{w}$ and $H_{x}^{0}=H_{z}^{0}=H_{w}^{0}$.

Finally, to show that $H_{x}^{0}$ is a group, we note that $[x, x] \in H_{x}^{0}$, that $[x, x][y, z]=[x y, x z]=[y, z]$ for all $[y, z] \in H_{x}^{0}$, and that if $[y, z] \in H_{x}^{0}$, then $[z, y] \in H_{x}^{0}$ and $[z, y][y, z]=[y z, y z]=[x, x]$. Also, it is obvious from 4.13 that each $H_{x}^{0}$ is closed under multiplication.

4.22. If $[y, z] \in H_{x}^{0}$ and $\left[y^{\prime}, z^{\prime}\right] \in H_{x^{\prime}}^{0}$, then $\left[y y^{\prime}, z z^{\prime}\right] \in H_{x x^{\prime}}^{0}$. Therefore the 1-to-1 mapping $H_{x} \rightarrow H_{x}^{0}$ is an isomorphism of $G^{\prime \prime}$ onto the semigroup $\left\{H_{x}^{0}\right\}$ with $H_{x}^{0} H_{x^{\prime}}^{0}=H_{x x^{\prime}}^{0}$. It is also easy to see that the mapping $x \rightarrow\left[x^{2}, x\right]$ is a 
1-to-1 mapping of $G^{\prime}$ onto a subsemigroup of $G^{0}$. This mapping is in fact an isomorphism of $G^{\prime}$ into $G^{0}$.

4.23. Theorem. Let $G$ be a commutative semigroup. Then $G$ has the property that $x^{2}=y^{2}=x y$ implies $x=y$ if and only if $G$ can be isomorphically imbedded in a semigroup that is the union of disjoint groups.

Proof. If $G$ has the property that $x^{2}=y^{2}=x y$ implies $x=y$, then $G$ is isomorphic to $G^{\prime}$, by 4.8 . Hence $G$ is isomorphic to a subsemigroup of $G^{0}$, by 4.22 . Conversely, if $G \subset U_{\imath} S_{\imath}$, where each $S_{\imath}$ is a commutative group, and if $x^{2}=y^{2}=x y$, where $x, y \in G$, then $x, x^{2}, y, y^{2}$, and $x y$ all lie in a single $S_{\iota}$. Since the cancellation law holds in $S_{\iota}$, we have $x=y$.

4.24. We summarize the constructions of the present section as follows. Starting with an arbitrary commutative semigroup $G$, we form a homomorphic image $G^{\prime}$ of $G$. The semigroup $G^{\prime}$ has the property that $x^{2}=y^{2}=x y$ implies $x=y$, and as will be shown later (6.2), $G^{\prime}$ is in a certain sense the largest semigroup with these properties. The semigroup $G^{\prime}$ is then imbedded in a semigroup $G^{0}$, which is the union of disjoint groups. The semigroup $G^{\prime \prime}$ is isomorphic to the subsemigroup of idempotent elements of $G^{0}$. The present $G^{0}$ is isomorphic to the semigroup $G^{0}$ of $[3,2.11]$, if $G$ is finite.

5. Necessary and sufficient conditions for semisimplicity of $l_{1}(G)$. In this section, we find a simple algebraic condition on $G$ equivalent to semisimplicity of $l_{1}(G)$ (Theorem 5.8). We also find the radical of $l_{1}(G)$ (Theorem 5.9). In doing these things, we first construct a number of semicharacters of $G$. We use the notation of $\$ 4$ and start by discussing semigroups $G^{\prime}$ as written in 4.9, that is, commutative semigroups $G^{\prime}$ such that $x^{2}=y^{2}=x y$ implies $x=y$, for all $x, y \in G^{\prime}$.

5.1. TheOREM. Let $\chi^{\prime}$ be a semicharacter of $G^{\prime}$. Then we have the following facts.

5.1.1. If $y \in H_{x}$, then $\chi^{\prime}(y)=0$ if and only if $\chi^{\prime}(x)=0$.

5.1.2. Let $\chi^{0}$ be defined by the relations

$$
\chi^{0}([x, y])=\left\{\begin{array}{l}
0 \text { if } \chi^{\prime}(x)=0, \\
\chi^{\prime}(x) / \chi^{\prime}(y) \text { if } \chi^{\prime}(x) \neq 0 .
\end{array}\right.
$$

Then $\chi^{0}$ is a multiplicative function on $G^{0}$ and $\chi^{0}$ is not identically 0 .

5.1.3. The function $\chi^{0}$ is a semicharacter of $G^{0}$ if and only if $\left|\chi^{\prime}(x)\right|=0$ or 1 for all $x \in G^{\prime}$.

5.1.4. We have $\chi^{0}\left(\left[x^{2}, x\right]\right)=\chi^{\prime}(x)$ for all $x \in G^{\prime}$.

Proof. Statement 5.1.1 follows directly from 4.10. Then 5.1.1 ensures that the definition of $\chi^{0}$ in 5.1.2 does not involve a zero in the denominator. If $[x, y]=[z, w]$, then $x w=y z$ and $z \in H_{x}$. This, together with 5.1.1 and the fact that $\chi^{\prime}$ is multiplicative, shows that $\chi^{0}$ is uniquely defined, i.e., that $\chi^{0}([x, y])$ $=\chi^{0}([z, w])$. We also have 
$\chi^{0}([x, y]) \chi^{0}([u, v])=\left\{\begin{array}{l}\frac{\chi^{\prime}(x)}{\chi^{\prime}(y)} \frac{\chi^{\prime}(u)}{\chi^{\prime}(v)}=\frac{\chi^{\prime}(x u)}{\chi^{\prime}(y v)}=\chi^{0}([x u, y v]) \text { if } \chi^{\prime}(x) \chi^{\prime}(u) \neq 0, \\ 0=\chi^{0}([x u, y v]) \text { if } \chi^{\prime}(x) \chi^{\prime}(u)=0 .\end{array}\right.$

This computation shows that $\chi^{0}$ is multiplicative. It is obvious that $\chi^{0}$ is not identically 0 and that 5.1.4 holds. Finally, to establish 5.1.3 we note first that if $\left|\chi^{\prime}(x)\right|=0$ or 1 , then the same is true of $\chi^{0}$, and therefore $\chi^{0}$ is a semicharacter. Conversely, suppose that $0<\left|\chi^{\prime}(y)\right|<1$ for some $y \in G^{\prime}$. Then $\left|\chi^{0}\left(\left[y, y^{n}\right]\right)\right|=\left|\chi^{\prime}(y)\right|^{1-n}$ is arbitrarily large for $n$ sufficiently large, and $\chi^{0}$ is in this case not a semicharacter.

5.2. ThEOREM. Let $\chi^{0}$ be a semicharacter of $G^{0}$ and let $\chi^{\prime}$ be the function on $G^{\prime}$ such that $\chi^{\prime}(x)=\chi^{0}\left(\left[x^{2}, x\right]\right)$ for all $x \in G^{\prime}$. Then we have the following facts.

5.2.1 The function $\chi^{\prime}$ is a semicharacter of $G^{\prime}$.

5.2.2 $\left|\chi^{\prime}(x)\right|=0$ or 1 for all $x \in G^{\prime}$.

$$
\chi^{0}([x, y])=\left\{\begin{array}{l}
0 \text { if } \chi^{\prime}(x)=0 \\
\chi^{\prime}(x) / \chi^{\prime}(y) \text { if } \chi^{\prime}(x) \neq 0 .
\end{array}\right.
$$

Proof. It is clear that $\chi^{\prime}$ is multiplicative and bounded, in view of 4.22. If $\chi^{0}([y, z]) \neq 0$, then $\chi^{\prime}(y)=\chi^{0}\left(\left[y^{2}, y\right]\right) \neq 0$, since $\left[y^{2}, y\right][y, y z]=[y, z]$. Therefore $\chi^{\prime}$ is a semicharacter of $G^{\prime}$. If $\chi^{\prime}(x) \neq 0$, then we have $\chi^{0}\left(\left[x^{2}, x\right]\right) \neq 0$. But we also have $\left[x^{2}, x\right]=[x, x]\left[x^{2}, x\right]$ and $\left[x, x^{2}\right]\left[x^{2}, x\right]=[x, x]$. From these equalities we find that $\chi^{0}([x, x])=1$ and $\chi^{0}\left(\left[x, x^{2}\right]\right)=\left(\chi^{0}\left(\left[x^{2}, x\right]\right)\right)^{-1}$. Since $\chi^{0}$ is bounded and $\left[x, x^{2}\right]^{n}$ and $\left[x^{2}, x\right]^{n}$ are elements of $G^{0}$ for all positive integers $n$, we infer that $\left|\chi^{\prime}(x)\right|=\left|\chi^{0}\left(\left[x^{2}, x\right]\right)\right|=1$. The verification of 5.2.3 is simple and is omitted.

5.3. Definition. If $G$ is a semigroup, let $\widehat{G}^{*}$ be the set of all semicharacters $\chi$ of $G$ such that $|\chi(x)|=0$ or 1 for all $x \in G$.

5.4. TheOREm. Let $G^{\prime}$ be as in the introduction to the present section. Then we have $\widehat{G}^{*} \cong \widehat{G}^{0}$.

Proof. This follows immediately from 5.1 and 5.2.

5.5. TheOREm. Let $u \in G^{\prime}$ and let $\chi_{u}^{0}$ be any character of the group $H_{u}^{0}$. Let

$$
\chi^{0}([x, y])=\left\{\begin{array}{l}
0 \text { if } H_{u} H_{x} \neq H_{u}, \\
\chi_{u}^{0}([u x, u y]) \text { if } H_{u} H_{x}=H_{u} .
\end{array}\right.
$$

Then $\chi_{u}^{0}$ is a semicharacter of $G^{0}$.

Proof. If $H_{u} H_{x}=H_{u}$, then $u x \in H_{u x}=H_{u} H_{x}=H_{u}$. Therefore $[u x, u y] \in H_{u}^{0}$ and $\chi^{0}([u x, u y])$ is defined. It is clear that $\chi^{0}$ is bounded and not identically 0 . We must still prove that $\chi^{0}([x, y]) \chi^{0}([z, w])=\chi^{0}([x z, y w])$. If $\chi^{0}([x z, y w]) \neq 0$, then $H_{u} H_{x z}=H_{u}$ and hence $H_{u} H_{x}=H_{u} H_{x z} H_{x}=H_{u} H_{x} H_{z}=H_{u}$ and $H_{u} H_{s}$ 
$=H_{u} H_{x} H_{z}=H_{u}$. We then have $\chi^{0}([x, y]) \chi^{0}([z, w])=\chi_{u}^{0}([u x, u y]) \chi_{u}^{0}([u z, u w])$ $=\chi_{u}^{0}([u x z, u y w])=\chi^{0}([x z, y w])$. If $\chi^{0}([x z, y w])=0$, then $H_{u} H_{x z} \neq H_{u}$. Since $H_{u} H_{x}=H_{u}$ and $H_{u} H_{z}=H_{u}$ imply $H_{u} H_{x z}=H_{u} H_{x} H_{z}=H_{u} H_{z}=H_{u}$, we have $\chi^{0}([x, y]) \chi^{0}([z, w])=0=\chi^{0}([x z, y w])$ in this case.

5.6. ThEOREM. The set $\widehat{G}^{*}$ is a separating family on $G^{\prime}$.

Proof. If $\chi^{0}$ is any of the semicharacters constructed in 5.5 and if $\chi^{\prime}(x)$ $=\chi^{0}\left(\left[x^{2}, x\right]\right)$, then obviously $\chi^{\prime} \in \widehat{G}^{\prime *}$. Suppose that $u \in G^{\prime}, v \in G^{\prime}$, and $u \neq v$. If $v \in H_{u}$, then $\left[v^{2}, v\right] \in H_{u}^{0}$ and the equality $\left[v^{2}, v\right]=\left[u^{2}, u\right]$ would imply $v^{2} u=v u^{2}$ and hence $v=u$, since the cancellation law holds in $H_{u}$ (4.15.1). Therefore if $v \in H_{u}$, there is a character $\chi_{u}^{0}$ of the commutative group $H_{u}^{0}$ such that $\chi_{u}^{0}\left(\left[v^{2}, v\right]\right) \neq \chi_{u}^{0}\left(\left[u^{2}, u\right]\right)$. (See for example $[9$, p. 94].) We use this $u$ and $\chi_{u}^{0}$ in 5.5.1 to obtain $\chi^{0}$, and obtain $\chi^{\prime}$ from $\chi^{0}$ by means of 5.2. We then have $\chi^{\prime}(v) \neq \chi^{\prime}(u)$. The other possibility is that $v \notin H_{u}$. If this is the case, we have either $H_{u} H_{v} \neq H_{u}$ or $H_{v} H_{u} \neq H_{v}$. If $H_{v} H_{u} \neq H_{v}$, we interchange $u$ and $v$ and can then suppose that $H_{u} H_{v} \neq H_{u}$. We use this $u$ and take $\chi_{u}^{0}$ identically 1 in 5.5. Then, by 5.2, we have $\chi^{\prime}(v)=0, \chi^{\prime}(u)=1$.

5.6.1. THEOREM. The sets $H_{x}$ are just the sets $U_{x}=\left\{y ; y \in G^{\prime}, \chi^{\prime}(y)=0\right.$ if and only if $\chi^{\prime}(x)=0$, for all $\left.\chi^{\prime} \in \widehat{G}^{\prime}\right\}$.

Proof. From 5.1.1, we see that $H_{x} \subset U_{x}$, for all $x \in G^{\prime}$. From the last part of the proof of 5.6, we see that $H_{x} \supset U_{x}$.

\subsubsection{ThEOREM. The algebra $l_{1}\left(G^{\prime}\right)$ is semisimple.}

Proof. This is a direct consequence of 3.5 and 5.6.

5.7. TheOREM. Let $G$ be a commutative semigroup. Then we have the following facts.

5.7.1. If $\chi$ is a semicharacter of $G$ and if $y \in T_{x}$, then $\chi(y)=\chi(x)$.

5.7.2. If $\chi$ is a semicharacter of $G$ and if $\chi^{\prime}\left(T_{x}\right)$ is defined as $\chi(x)$, then $\chi^{\prime}$ is a semicharacter of $G^{\prime}$.

5.7.3. If $\chi^{\prime}$ is a semicharacter of $G^{\prime}$ and if $\chi(x)$ is defined as $\chi^{\prime}\left(T_{x}\right)$, then $\chi$ is a semicharacter of $G$.

Proof. To prove 5.7.1, let $y$ be any element of $T_{x}$. By 4.1, we have $x y^{n}$ $=y^{n+1}, y x^{n}=x^{n+1}$ for some positive integer $n$. Therefore we have $\chi(x) \chi(y)^{n}$ $=\chi(y)^{n+1}$ and $\chi(y) \chi(x)^{n}=\chi(x)^{n+1}$. It follows that $\chi(x) \chi(y)=\chi(y)^{2}=\chi(x)^{2}$. This in turn implies that $\chi(y)=\chi(x)$ and 5.7.1 follows.

The function $\chi^{\prime}$ defined in 5.7.2 is single-valued because of 5.7.1. The function $\chi^{\prime}$ is clearly bounded and not identically 0 . It is multiplicative in view of 4.4. The proof of 5.7.3 is equally simple and is omitted.

5.8. ThEOREM. Let $G$ be a commutative semigroup. Then $l_{1}(G)$ is semisimple if and only if, for all $x, y \in G, x^{2}=y^{2}=x y$ implies $x=y$. 
Proof. The necessity of the condition stated follows from 5.7.1 and 3.5. The sufficiency follows from 5.6.2, 4.8, and 4.4.

5.9. TheOREM. The radical $\beta$ of $l_{1}(G)$ consists of all $A=\sum_{x \in G} \alpha(x) \lambda_{x}$, $\sum_{x \in G}|\alpha(x)|<\infty$, such that $\sum_{x \in T} \alpha(x)=0$ for all $T \in G^{\prime}$.

Proof. If $\sum_{x \in T} \alpha(x)=0$ for all $T \in G^{\prime}$, then, by 5.7.1, we have $\sum_{x \in G} \alpha(x) \chi(x)=\sum_{T \in G^{\prime}} \sum_{x \in T} \alpha(x) \chi(x)=0$ for all $\chi \in \widehat{G}$. Therefore, by 2.8, we have $A \in \mathcal{P}$.

Conversely, if $A \in P$, then, by 2.8 , we have $\sum_{x \in G} \alpha(x) \chi(x)=0$ for all $\chi \in \widehat{G}$. By 5.7.2, we have $0=\sum_{T \in G^{\prime}} \sum_{x \in T} \alpha(x) \chi(x)=\sum_{T \in G^{\prime}}\left\{\sum_{x \in T} \alpha(x)\right\}$ $\chi^{\prime}(T)$, for all $\chi^{\prime} \in \widehat{G^{\prime}}$. Using 5.6.2 and 2.8, we now obtain the fact that $\sum_{x \in T} \alpha(x)=0$ for all $T \in G^{\prime}$.

5.9.1. Corollary. The difference algebra $l_{1}(G)-\odot$ is isomorphic to $l_{1}\left(G^{\prime}\right)$.

\section{Miscellaneous facts about semicharacters.}

6.1. By way of summary, we now review the constructions of $\$ \$ 4$ and 5 relating to the structure of $G$ and the semicharacters of $G$. Let $G$, then, be an arbitrary commutative semigroup. The mapping $x \rightarrow T_{x}$ is a homomorphism of $G$ onto $G^{\prime}$ : we denote the element $T_{x}$ of $G^{\prime}$ by the symbol $x^{\prime}$. The mapping $x^{\prime} \rightarrow H_{x^{\prime}}$ is a homomorphism of $G^{\prime}$ onto the idempotent semigroup $G^{\prime \prime}$ : we denote the element $H_{x^{\prime}}$ of $G^{\prime \prime}$ by the symbol $x^{\prime \prime}$. The subsemigroups $H_{x^{\prime}}$ have interesting properties. Either they are groups or they contain no idempotent elements; no semicharacter on $H_{x^{\prime}}$ vanishes anywhere; they obey the cancellation law. In a sense they are the elementary building blocks from which $G^{\prime}$ is constructed, and $G^{\prime \prime}$ is the framework on which these blocks are put. It is clear that all semigroups $G^{\prime}$ can be characterized in terms of the semigroup $G^{\prime \prime}$, the semigroups $H_{x^{\prime}}$, and a suitable family of homomorphisms. (See [1, Theorem 3], where this is done in a somewhat similar case.)

The semigroup $G^{0}$ can be thought of as containing $G^{\prime}$ : in it, all of the semigroups $H_{x^{\prime}}$ are imbedded in groups. For $x \in G$, let $x^{0}$ denote the element $\left[x^{\prime 2}, x^{\prime}\right]$ of $G^{0}$. Other elements of $G^{0}$ may be denoted by $y^{*}, z^{*}, \ldots$. For an example of different ways to imbed a certain $G$ in a union of groups, see 11.3.

It is plain that $\widehat{G} \cong \widehat{G^{\prime}}$; that $\widehat{G^{\prime \prime}}$ is isomorphic to the set of all semicharacters of $G$ and $G^{\prime}$ assuming only the values 0 and 1 ; and that $\widehat{G}^{*} \cong \widehat{G}^{*} \cong \widehat{G^{0}}$.

The following three theorems hardly require formal proofs.

6.2. TheOREM. Let $G$ be a commutative semigroup, and let $H$ be a homomorphic image of $G$ such that $T_{x}=\{x\}$ for all $x \in H$. Then $H$ is a homomorphic image of $G^{\prime}$.

6.3. TheOREM. Let $G$ be as in 6.2 and let $H$ be an idempotent commutative semigroup that is a homomorphic image of $G$. Then $H$ is a homomorphic image of $G^{\prime \prime}$. 
6.4. THEOREM. Let $G$ be an arbitrary semigroup containing a subsemigroup $S$ whose complement $T$ is an ideal. Let $\psi$ be any semicharacter of $S$, and let $\chi$ be the function on $G$ such that $\chi(x)=\psi(x)$ for all $x \in S, \chi(x)=0$ for all $x \in T$. Then $\chi$ is a semicharacter of $G$.

The following three theorems will be useful in the sequel. In all of them, $G$ is an arbitrary commutative semigroup.

6.5. TheOREM. Let $y \in G^{\prime}$ and let $\psi$ be a semicharacter of $H_{y}$. Then the function $\chi^{\prime}$, defined by the relations

$$
\chi^{\prime}(x)=\left\{\begin{array}{l}
0 \text { if } H_{y} H_{x} \neq H_{y} \\
\frac{\psi(y x)}{\psi(y)} \text { if } H_{y} H_{x}=H_{y}
\end{array}\right.
$$

is a semicharacter of $G^{\prime}$.

Proof. Since $\psi$ is not identically 0 , we have $\psi(y) \neq 0$ from 4.14. If $H_{y} H_{x}$ $=H_{y}$, then $y x \in H_{y}$ and we see that $\chi^{\prime}(x)$ is defined for all $x \in G^{\prime}$. It is clear that $\chi^{\prime}$ is bounded and not identically 0 on $G^{\prime}$. Furthermore, for $x \in G^{\prime}$ and $z \in G^{\prime}$, we have

$$
\chi^{\prime}(x) \chi^{\prime}(z)=\left\{\begin{array}{l}
\frac{\psi(y x)}{\psi(y)} \frac{\psi(y z)}{\psi(y)}=\frac{\psi(y) \psi(y x z)}{\psi(y) \psi(y)}=\frac{\psi(x y z)}{\psi(y)} \text { if } H_{y} H_{x}=H_{y} H_{z}=H_{y}, \\
0 \text { otherwise. }
\end{array}\right.
$$

We also have

$$
\chi^{\prime}(x z)=\left\{\begin{array}{l}
\frac{\psi(y x z)}{\psi(y)} \text { if } H_{y} H_{x z}=H_{y}, \\
0 \text { if } H_{y} H_{x z} \neq H_{y} .
\end{array}\right.
$$

As in the proof of 5.5, we see that $H_{y} H_{x}=H_{y} H_{z}=H_{y}$ if and only if $H_{y} H_{x z}=H_{y}$. Therefore $\chi^{\prime}(x) \chi^{\prime}(z)=\chi^{\prime}(x z)$.

6.6. Theorem. For $w \in G$, let $w^{\prime}=T_{w} \in G^{\prime}$ and $w^{\prime \prime}=H_{w^{\prime}} \in G^{\prime \prime}$. (A) Let $\chi$ be a semicharacter of $G$ and let $\chi^{\prime \prime}$ on $G^{\prime \prime}$ be defined by

$$
\chi^{\prime \prime}\left(w^{\prime \prime}\right)= \begin{cases}1 & \text { if } \chi(w) \neq 0 \\ 0 & \text { if } \chi(w)=0\end{cases}
$$

Then $\chi^{\prime \prime}$ is a semicharacter of $G^{\prime \prime}$. (B) If $\chi^{\prime \prime}$ is a semicharacter of $G^{\prime \prime}$, let $\chi$ be the function on $G$ such that $\chi(w)=\chi^{\prime \prime}\left(w^{\prime \prime}\right)$ for all $w \in G$. Then $\chi$ is a semicharacter of $G$.

Proof. (A) If $w_{1}^{\prime \prime}=w_{2}^{\prime \prime}$, then $w_{2}^{\prime} \in H_{w_{1}^{\prime}}$ and, for any $\chi^{\prime} \in \widehat{G}^{\prime}, \chi^{\prime}\left(w_{1}^{\prime}\right)$ and $\chi^{\prime}\left(w_{2}^{\prime}\right)$ are both 0 or both different from 0 , by 5.1.1. Then 5.7 shows that $\chi\left(w_{1}\right)$ and $\chi\left(w_{2}\right)$ are both 0 or both different from 0 for all $\chi \in \widehat{G}$. Thus $\chi^{\prime \prime}$ is 
well defined. It is clearly bounded, not identically 0 , and multiplicative.

(B) The function $\chi$ assumes only the values 0 and 1 , and is not identically 0 . It is seen by 4.3 and 4.13 to be multiplicative.

6.7. THEOREM. $\widehat{G}$ is a semigroup if and only if for all $w_{1}^{\prime \prime}$ and $w_{2}^{\prime \prime}$ in $G^{\prime \prime}$, there exists a $w_{3}^{\prime \prime}$ in $G^{\prime \prime}$ such that $w_{3}^{\prime \prime} w_{1}^{\prime \prime}=w_{1}^{\prime \prime}$ and $w_{3}^{\prime \prime} w_{2}^{\prime \prime}=w_{2}^{\prime \prime}$.

Proof. We use the notation of 6.6. Since $\widehat{G}$ is a semigroup if and only if $\chi_{1} \chi_{2}$ is not identically 0 for any $\chi_{1}$ and $\chi_{2}$ in $\widehat{G}$, we see from 6.6 that $\widehat{G}$ is a semigroup if and only if $\chi_{1}^{\prime \prime} \chi_{2}^{\prime \prime}$ is not identically 0 for any $\chi_{1}^{\prime \prime}$ and $\chi_{2}^{\prime \prime}$ in $\widehat{G^{\prime \prime}}$.

Now we have $\chi_{1}^{\prime \prime}\left(w_{1}^{\prime \prime}\right) \neq 0$ and $\chi_{2}^{\prime \prime}\left(w_{2}^{\prime \prime}\right) \neq 0$ for some $w_{1}^{\prime \prime}$ and $w_{2}^{\prime \prime}$ in $G^{\prime \prime}$. If $w_{3}^{\prime \prime} w_{1}^{\prime \prime}=w_{1}^{\prime \prime}$ and $w_{3}^{\prime \prime} w_{2}^{\prime \prime}=w_{2}^{\prime \prime}$ for some $w_{3}^{\prime \prime}$ in $G^{\prime \prime}$, then $\chi_{1}^{\prime \prime}\left(w_{1}^{\prime \prime}\right) \chi_{2}^{\prime \prime}\left(w_{2}^{\prime \prime}\right)$ $=\chi_{1}^{\prime \prime}\left(w_{3}^{\prime \prime} w_{1}^{\prime \prime}\right) \chi_{2}^{\prime \prime}\left(w_{3}^{\prime \prime} w_{2}^{\prime \prime}\right)=\chi_{1}^{\prime \prime}\left(w_{3}^{\prime \prime}\right) \chi_{2}^{\prime \prime}\left(w_{3}^{\prime \prime}\right) \chi_{1}^{\prime \prime}\left(w_{1}^{\prime \prime}\right) \chi_{2}^{\prime \prime}\left(w_{2}^{\prime \prime}\right)$, and hence $\chi_{1}^{\prime \prime}\left(w_{3}^{\prime \prime}\right) \chi_{2}^{\prime \prime}\left(w_{3}^{\prime \prime}\right)=1$. Thus $\chi_{1}^{\prime \prime} \chi_{2}^{\prime \prime}$ is not identically 0 .

Conversely, if there exist $w_{1}^{\prime \prime}$ and $w_{2}^{\prime \prime}$ in $G^{\prime \prime}$ such that there is no $w_{3}^{\prime \prime}$ for which $w_{3}^{\prime \prime} w_{1}^{\prime \prime}=w_{1}^{\prime \prime}$ and $w_{3}^{\prime \prime} w_{2}^{\prime \prime}=w_{2}^{\prime \prime}$, then the functions $\chi_{i}^{\prime \prime}$ such that $\chi_{i}^{\prime \prime}\left(w^{\prime \prime}\right)=1$ if $w_{i}^{\prime \prime} w^{\prime \prime}=w_{i}^{\prime \prime}, \chi_{i}^{\prime \prime}\left(w^{\prime \prime}\right)=0$ otherwise, $i=1,2, w^{\prime \prime} \in G^{\prime \prime}$, are semicharacters of $G^{\prime \prime}$ and $\chi_{1}^{\prime \prime} \chi_{2}^{\prime \prime}=0$.

7. The unit of $l_{1}(G)$. We here give conditions on a commutative semigroup that are necessary and sufficient for the existence of a unit in $l_{1}(G)$.

7.1. Definition. Let $G$ be a semigroup, not necessarily commutative. A set $A \subset G$ is said to be a set of relative units for $G$ if, for every $x \in G$, there exists $a \in A$ such that $x a=a x=x$.

For use in $\S 8$, we note the following fact.

7.2. TheоRem. Let $G$ be a semigroup, not necessarily commutative, and suppose that $G$ has a finite set $A=\left\{a_{1}, a_{2}, \cdots, a_{m}\right\}$ of relative units. Then there exists a subset $B$ of $A$ such that $B$ is a set of relative units for $G$, all elements of $B$ are idempotent, and no proper subset of $B$ is a set of relative units for $G$.

Proof. Consider an arbitrary element of $A$, say $a_{1}$. Since $A$ is a set of relative units for $G$, there exists an integer $j, 1 \leqq j \leqq m$, such that $a_{1} a_{j}=a_{j} a_{1}=a_{1}$. If there exists a $j>1$ with this property, then $\left\{a_{2}, a_{3}, \cdots, a_{m}\right\}$ is a set of relative units for $G$. To see this, let $x \in G$ and let $a_{1} x=x a_{1}=x$. Then $a_{j} x=a_{j}\left(a_{1} x\right)$ $=\left(a_{j} a_{1}\right) x=a_{1} x=x$, and similarly $x a_{j}=x$. Since $A$ is finite, we may repeat this argument a finite number of times to produce a subset $B$ of $A$, which we write as $\left\{b_{1}, b_{2}, \cdots, b_{n}\right\}(n \leqq m)$ with the properties that $B$ is a set of relative units for $G$ and if $b_{i}, b_{j} \in B$ and $b_{i} b_{j}=b_{j} b_{i}=b_{i}$, then $b_{i}=b_{j}$. This completes the proof.

7.3. TheOREM. Let $G$ be a commutative semigroup. If $l_{1}(G)$ has a unit, then $G$ contains a finite set of relative units.

Proof. Let $E=\sum_{u \in G} \epsilon_{u} \lambda_{u}, \sum_{u \in G}\left|\epsilon_{u}\right|<\infty$, be the unit of $l_{1}(G)$. Let the set $\left\{u ; u \in G, \epsilon_{u} \neq 0\right\}$ be written as $\left\{u_{1}, u_{2}, u_{3}, \cdots, u_{n}, \cdots\right\}$, and for convenience, write $\epsilon_{u_{j}}$ as $\epsilon_{j}(j=1,2,3, \cdots)$. Let $m$ be a positive integer such that 
$\sum_{j=m+1}^{\infty}\left|\epsilon_{j}\right|<1$. For all $x \in G$, we have $E * \lambda_{x}=\lambda_{x}$, and since $E * \lambda_{x}=\sum_{j=1}^{\infty} \epsilon_{j} \lambda_{u_{j} x}$, we have

7.3 .1

$$
1=\sum_{i, u_{j} x=x} \epsilon_{j}=\sum_{1 \leqq j \leqq m, u_{j} x=x} \epsilon_{j}+\sum_{m<j<\infty, u_{j} x=x} \epsilon_{j} .
$$

The last summand in 7.3.1 is in absolute value less than 1 , and hence there is an integer $j$ such that $1 \leqq j \leqq m$ and $u_{j} x=x$. Since $G$ is commutative, we have shown that $\left\{u_{1}, u_{2}, \cdots, u_{m}\right\}$ is a finite set of relative units for $G$.

7.4. Note. With an obvious definition of relative right and left units in $G, 7.3$ has an obvious analogue for the cases in which $G$ is not commutative and $\mathrm{l}_{1}(G)$ has a right or left unit.

7.5. Theorem. Let $G$ be a commutative semigroup containing a finite set of relative units. Then $l_{1}(G)$ has a unit.

Proof. Let $\left\{a_{1}, a_{2}, \cdots, a_{m}\right\}$ be a finite set of relative units for $G$. We may suppose without loss of generality that $a_{1}, a_{2}, \cdots, a_{m}$ are idempotent (7.2). Let $E \in l_{1}(G)$ be defined by

$$
\begin{aligned}
E= & \sum_{1 \leqq i \leqq m} \lambda_{a_{i}}-\sum_{1 \leqq i<j \leqq m} \lambda_{a_{i} a_{j}} \\
& +\sum_{1 \leqq i<j<k \leqq m} \lambda_{a_{i} a_{a} a_{k}}-\cdots+(-1)^{m+1} \lambda_{a_{1} a_{2}} \cdots a_{m}
\end{aligned}
$$

It is easy to see that $E$ is a unit for $l_{1}(G)$.

7.6. Note. If $G$ has a unit $e$, it is obvious that $\lambda_{e}$ is the unit of $l_{1}(G)$. Simple examples, as in 11.1.6 for instance, show that $l_{1}(G)$ can have a unit even if $G$ does not. The unit of $l_{1}(G)$, if it exists, must be a finite linear combination of functionals $\lambda_{x}$ with idempotent $x$, as 7.5.1 shows. Since considerable cancellation can take place in the right side of 7.5.1, the unit of $l_{1}(G)$ may be much simpler in appearance than 7.5 .1 indicates.

\section{Topology of $\widehat{G}$.}

8.1. Throughout this section, $G$ is a commutative semigroup. According to the well-known Gel'fand theory for commutative Banach algebras (see for example [5, Chapters IV and V]), the space of regular maximal ideals of $l_{1}(G)$ may be identified with the space of all homomorphisms of $l_{1}(G)$ onto the complex number field $[5$, p. 69 , Theorem $]$. By 2.7 , we can therefore identify the space of regular maximal ideals of $l_{1}(G)$ with the set $\widehat{G}$.

In the usual way, we associate with every $A=\sum_{x \in G} \alpha(x) \lambda_{x} \in l_{1}(G)$ its Fourier transform $\widehat{A}$, which is the complex function on $\widehat{G}$ whose value at $\chi \in \widehat{G}$ is

\section{1 .1}

$$
A(\chi)=\sum_{x \in G} \alpha(x) \chi(x) .
$$

The Gel'fand topology of $\widehat{G}$ is the weakest topology (i.e., the topology with 
the smallest family of open sets) under which all of the functions $\widehat{A}$ are continuous. Throughout the remainder of the present section, except where the contrary is explicitly stated, we shall take $\widehat{G}$ to be a topological space with the Gel'fand topology. The product of 2 elements of $\widehat{G}$ is defined as in $[3,3.7]$. It is well known that $\widehat{G}$ is a locally compact Hausdorff space, that all functions $\widehat{A}$ vanish at infinity in $\widehat{G}$, and that $\widehat{G}$ is compact if $l_{1}(G)$ has a unit.

8.2. Theorem. Let $\chi$ be an element of $\widehat{G}$, let $\left\{x_{1}, x_{2}, \cdots, x_{m}\right\}$ be a finite subset of $G$, and $\delta$ a positive real number. Let $U_{x_{1}}, \ldots, x_{m} ; \delta(\chi)$ be the set of all $\psi \in \widehat{G}$ such that $\left|\psi\left(x_{j}\right)-\chi\left(x_{j}\right)\right|<\delta(j=1, \cdots, m)$. Each set $U_{x_{1}, \cdots, x_{m} ;}(\chi)$ is an open neighborhood of $\chi$, and the set of all of these neighborhoods is a complete system of neighborhoods for $\chi$.

We omit the proof of this simple result.

8.3. Remark. We may restate the idea of 8.2 as follows. Let $D$ be the multiplicative semigroup $\{z ; z$ is a complex number, $|z| \leqq 1\}$ with the ordinary topology. For a cardinal number $\mathfrak{m}>0$ (finite or infinite), let $D^{\mathfrak{m}}$ be the Cartesian product of $\mathrm{m}$ replicas of $D$. Under the usual Cartesian product topology, $D^{\mathfrak{m}}$ is a compact Hausdorff space. With multiplication defined coordinate-wise, $D^{\mathfrak{m}}$ becomes a compact semigroup in which multiplication is continuous in both variables. The set $\mathfrak{B}_{1}$ of all complex-valued functions $f$ on $G$ such that $|f(x)| \leqq 1$ for all $x \in G$ can be identified with the semigroup $D^{\mathfrak{m}}$ such that $\mathfrak{m}$ is the cardinal number of $G$. Every element of $\widehat{G}$ is an element of $\mathfrak{B}_{1}$. Then 8.2 asserts that the Gel'fand topology of $\widehat{G}$ is the relative topology of $\widehat{G}$ as a subspace of $\mathfrak{B}_{1}$, where $\mathfrak{B}_{1}$ has the Cartesian product topology of a $D^{\mathfrak{m}}$. We have also the following facts, which are easily verified.

8.4. Theorem. Let 0 denote the function in $\mathfrak{B}_{1}$ that is identically 0 . Then $\widehat{G} \cup\{0\}$ is a compact subsemigroup of $\mathfrak{B}_{1}$.

8.5. THEOREM. $\widehat{G}$ is compact if and only if 0 is not a limit point of $\widehat{G}$ in $\mathfrak{B}_{1}$.

8.6. TheOREM. The mapping $\Delta$ defined by $\chi \rightarrow \chi^{\prime}$, where $\chi^{\prime}\left(T_{x}\right)=\chi(x)$ (see 5.7) is a homeomorphism as well as an isomorphism of $\widehat{G}$ onto $\widehat{G^{\prime}}$.

8.7. TheOREM. If $l_{1}\left(G^{\prime}\right)$ has a unit, then $\widehat{G}$ is compact.

Proof. As remarked in 8.1 , if $l_{1}\left(G^{\prime}\right)$ has a unit, then $\widehat{G}^{\prime}$ is compact. Now apply 8.6.

The converse of 8.7 is also true, but its proof is rather long. We break up the proof into several steps.

8.8. ThEOREM. If $\widehat{G}$ is compact, then $G^{\prime \prime}$ has a finite set of relative units.

Proof. Like every idempotent commutative semigroup (see $[3,2.7]$ ), $G^{\prime \prime}$ is a semilattice under the partial ordering $H_{u} \leqq H_{v}$ if and only if $H_{u} H_{v}=H_{u}$. Now let $\Gamma$ be an arbitrary ordinal number and suppose that $\left\{H_{u_{\gamma}}\right\}_{\gamma<r}$ is a subset of $G^{\prime \prime}$ such that $\gamma<\delta<\Gamma$ implies $H_{u_{\gamma}} \leqq H_{u_{\delta}}$ and $H_{u_{\gamma}} \neq H_{u_{8}}$. Suppose 
that there is no $H_{v} \in G^{\prime \prime}$ such that $H_{v} \geqq H_{u_{\gamma}}$ for all $\gamma<\Gamma$. Then let $\chi_{\gamma}$ be the semicharacter of $G$ defined by 5.5 .1 with $u=u_{\gamma}$ and $\chi_{u_{\gamma}}^{0}$ identically 1 on $H_{u_{\gamma}}$. Now let $\left\{x_{1}, x_{2}, \cdots, x_{m}\right\}$ be an arbitrary finite subset of $G$. Under our hypothesis, there exists for every $i, i=1,2, \cdots, m$, an ordinal number $\gamma(i)<\Gamma$ such that $H_{u_{\gamma(i)}} H_{x_{i}} \neq H_{u_{\gamma(i)}}$. Let $\delta=\max \{\gamma(1), \gamma(2), \cdots, \gamma(m)\}$. Then by the definition of the semicharacters $\chi_{\gamma}$, it follows that $\chi_{\delta}\left(x_{i}\right)=0$ for $i=1,2, \cdots, m$. Therefore every neighborhood of 0 in $\mathfrak{B}_{1}$ contains a semicharacter, and by $8.5, \widehat{G}$ is noncompact. We have therefore proved the following fact.

8.8.1. If $\widehat{G}$ is compact, then every well-ordered increasing transfinite sequence of elements of $G^{\prime \prime}$ admits an upper bound in $G^{\prime \prime}$.

It is a simple consequence of 8.8.1 (we omit the proof) that $G^{\prime \prime}$ contains elements that are maximal in the ordering $\leqq$. In fact, for every $H_{x} \in G^{\prime \prime}$, there exists $H_{a} \in G^{\prime \prime}$ such that $H_{a}$ is maximal and $H_{a} \geqq H_{x}$. The reader will note that the elements here called maximal are those called prime in 3.8 of [3].

We next show that $G^{\prime \prime}$ contains only a finite number of maximal elements. Supposing the contrary, let $\left\{H_{a_{1}}, H_{a_{2}}, \cdots, H_{a_{n}}, \cdots\right\}$ be a countably infinite set of distinct maximal elements of $G^{\prime \prime}$. For $n=1,2,3, \cdots$, let $\chi_{n}$ be the function on $G$ such that

$$
\chi_{n}(x)= \begin{cases}1 & \text { if } x \in H_{a_{n}} \\ 0 & \text { otherwise. }\end{cases}
$$

Theorem 5.5, 5.2.1, and the maximality of $H_{a_{n}}$ show that $\chi_{n}$ is a semicharacter of $G$. If now $\left\{x_{1}, x_{2}, \cdots, x_{m}\right\}$ is an arbitrary finite subset of $G$, there are clearly an infinite number of positive integers $n$ such that $\left\{x_{1}, x_{2}, \cdots, x_{m}\right\}$ $\cap H_{a_{n}}=0$. For every such $n$, we have $\chi_{n}\left(x_{i}\right)=0(i=1,2, \cdots, m)$, and therefore every neighborhood in $\mathfrak{B}_{1}$ of the function 0 contains points of $\widehat{G}$. Theorem 8.5 shows that $\widehat{G}$ is not compact. We thus have the following fact.

8.8.2. If $\widehat{G}$ is compact, then $G^{\prime \prime}$ contains only a finite number of maximal elements.

Using 8.8.1 and 8.8.2, we can quickly complete the proof. From 8.8.1, we know that $G^{\prime \prime}$ contains a set $\left\{H_{a}\right\}$ of maximal elements such that for all $H_{x} \in G^{\prime \prime}$, there exists an $H_{a}$ for which $H_{a} H_{x}=H_{x}$. By 8.8.2, the set $\left\{H_{a}\right\}$ is finite, and is therefore a finite set of relative units for $G^{\prime \prime}$. (Note that this set automatically satisfies the conditions imposed on the set $B$ in 7.2.) This completes the proof of 8.8 .

\subsubsection{CoRollary. If $\widehat{G}$ is compact, then $l_{1}\left(G^{\prime \prime}\right)$ has a unit.}

Simple examples (see 11.4) show that the converse of 8.8 .3 is false. We next turn our attention to a special case, that of semigroups in which $T_{x}=\{x\}$ and in which there is only one set $H_{x}$. In this case, we obtain as by-products some interesting facts about such semigroups. 
8.9. Theorem. Let $G$ be a commutative semigroup such that $T_{x}=\{x\}$ for all $x \in G$ and such that for all $x, y \in G$, there exist $u, v \in G$ and a positive integer $n$ for which $x^{n}=u y$ and $y^{n}=v x$. Then $\widehat{G}$ is compact if and only if $G$ is a group.

Proof. Since $T_{x}=\{x\}$ for all $x \in G$, we have $G \cong G^{\prime}$, and by 5.6.2, $l_{1}(G)$ is semisimple. Now let $x$ be an arbitrary element of $G$. We have $\widehat{\lambda}_{x}(\chi)=\chi(x)$ for all $\chi \in \widehat{G}$. It follows from 5.1.1 and the fact that $G$ is a single $H_{x}$ that $\chi$ vanishes nowhere on $G$. In other words, the function $\widehat{\lambda}_{x}$ vanishes nowhere on $\widehat{G}$. Therefore $l_{1}(G)$ is a semisimple commutative Banach algebra containing an element whose Fourier transform vanishes nowhere. If $\widehat{G}$ is compact, it is known that $l_{1}(G)$ must in this case contain a unit [5, p. 80, Corollary]. Since $l_{1}(G)$ contains a unit, $G$ contains a finite set of relative units (7.3). These relative units may be taken as idempotents (7.2). Therefore $G$ contains an idempotent, and by $4.16, G$ must be a group.

Conversely, if $G$ is a group, then $G$ contains a unit and $l_{1}(G)$ contains a unit. Therefore $\widehat{G}$ is compact. This completes the proof.

8.10. THEOREM. Let $G$ be a semigroup satisfying the hypotheses of 8.9. Then $G$ is a non-group if and only if $\widehat{G}$ contains an element $\chi$ such that $0<|\chi(x)|<1$ for all $x \in G$.

Proof. Since every semicharacter of a group is a character, no semicharacter of $G$ can have absolute value less than 1 at any point if $G$ is a group. Conversely, suppose that $G$ is a non-group. By $8.9, \widehat{G}$ is noncompact. In view of 8.5 , this implies that for every finite subset $\left\{x_{1}, x_{2}, \cdots, x_{m}\right\}$ of $G$ and every positive real number $\delta$, there exists an element $\chi \in \widehat{G}$ such that $\left|\chi\left(x_{j}\right)\right|<\delta$ $(j=1,2, \cdots, m)$. It follows a fortiori that $\widehat{G}$ contains a semicharacter $\chi$ whose absolute value is at some point less than 1 . Now if there existed an element $y \in G$ such that $|\chi(y)|=1$, we could define a function $\psi$ on $G$ as follows: $\psi(u)=1$ if $|\chi(u)|=1, \psi(u)=0$ if $|\chi(u)|<1$. It is easy to see that $\psi$ would be a semicharacter of $G$ assuming the values 0 and 1 and no other values. This contradicts 5.1.1, and so we have $|\chi(x)|<1$ for all $x \in G$. This completes the proof.

From 8.10 , we easily obtain the following fact.

8.11. Theorem. Let $G$ be a commutative semigroup. The following conditions on $G$ are equivalent:

8.11.1 $\widehat{G}^{*}=\widehat{G}$;

8.11.2 $G^{\prime}$ is a union of disjoint groups;

8.11.3 every subsemigroup $H_{x}$ of $G^{\prime}$ is a group;

8.11.4 $G^{\prime} \cong G^{0}$.

Proof. The logical equivalence of 8.11.2, 8.11.3, and 8.11.4 is simple to establish: we omit the proof. Suppose then that 8.11.1 fails, and that $\chi$ is a semicharacter of $G$ such that $0<|\chi(u)|<1$ for some $u \in G$. Then we have $0<\left|\chi^{\prime}\left(T_{u}\right)\right|<1$, where $\chi^{\prime}$ is defined as in 5.7.2. Now, changing our notation 
in mid-proof, write the elements of $G^{\prime}$ as $x, y, z, \cdots$. We see that there exists an $x \in G^{\prime}$ and a semicharacter $\chi^{\prime}$ of $G^{\prime}$ such that $0<\left|\chi^{\prime}(x)\right|<1$. Theorem 8.10 implies that the subsemigroup $H_{x}$ is a non-group. Hence 8.11 .3 implies 8.11.1.

Suppose next that 8.11.3 fails. Then there is a $u \in G^{\prime}$ such that $H_{u}$ is a nongroup, and, by 8.10 , there is a semicharacter $\chi_{u}$ of $H_{u}$ such that $0<\left|\chi_{u}(z)\right|$ $<1$ for all $z \in H_{u}$. By the construction given in $6.5, \chi_{u}$ admits an extension over $G^{\prime}$, which we write as $\chi^{\prime}$. The semicharacter $\chi$ of $G$ corresponding to $\chi^{\prime}$ under the correspondence described in 5.7 is clearly a semicharacter of $G$ that assumes a nonzero value less than 1 in absolute value. Hence 8.11.1 implies 8.11.3, and the proof is complete.

After this digression, we return to our main line of investigation.

8.12. THEOREM. Let $G$ be a commutative semigroup. If $\widehat{G}$ is compact, then $l_{1}\left(G^{\prime}\right)$ has a unit.

Proof. If $\widehat{G}$ is compact, then $G^{\prime \prime}$ contains a finite set $\left\{H_{a_{1}}, H_{a_{2}}, \cdots, H_{a_{n}}\right\}$ of maximal elements that are relative units for $G^{\prime \prime}$. We shall show that each $H_{a_{j}}$ is a group if $\widehat{G}$ is compact. Suppose that some $H_{a_{j}}$ is a non-group. Then, by 8.10, there is a semicharacter $\chi_{j}$ of $H_{a_{j}}$ such that $0<\left|\chi_{j}(x)\right|<1$ for all $x \in H_{a_{j}}$. Let $\chi$ be the function on $G^{\prime}$ such that $\chi(x)=\chi_{j}(x)$ for $x \in H_{a_{j}}$ and $\chi(x)=0$ for $x \notin H_{a_{j}}$. It follows from 6.5 that $\chi \in \widehat{G^{\prime}}$. Note that we have $|\chi(x)|<1$ for all $x \in G^{\prime}$. Let $\left\{x_{1}, x_{2}, \cdots, x_{m}\right\}$ be an arbitrary finite subset of ' $G^{\prime}$ and $\delta$ an arbitrary positive real number. It is clear that there exists a positive integer $p$ such that $\left|\chi^{p}\left(x_{j}\right)\right|<\delta$ for $j=1,2, \cdots, m$; that is, 0 is a limit point in $\mathfrak{B}_{1}$ of. $\widehat{G^{\prime}}$. Therefore $\widehat{G}^{\prime}$ is noncompact, by 8.5 . By $8.6, \widehat{G}$ is noncompact. Therefore, if $\widehat{G}$ is compact, every $H_{a_{j}}$ is a group. It is a notational matter only to suppose that $a_{j}$ is the unit of $H_{a_{j}}(j=1,2, \cdots, n)$. Let the element $\sum_{j=1}^{n} \lambda_{a_{j}}$ of $l_{1}\left(G^{\prime}\right)$ be written as $A$. It is clear that $\widehat{A}(\chi) \geqq 1$ for all $\chi \in \widehat{G^{\prime}}$. Hence the maximal ideal space of $l_{1}\left(G^{\prime}\right)$ is compact, $l_{1}\left(G^{\prime}\right)$ is semisimple, and $l_{1}\left(G^{\prime}\right)$ contains an element whose Fourier transform vanishes nowhere. Hence, by $[5$, p. 80 , Corollary $], l_{1}\left(G^{\prime}\right)$ has a unit.

8.13. REMARK. The foregoing proof can be completed without recourse to [5, p. 80 , Corollary], as follows. Given $x \in G^{\prime}$, there is an $a$, such that $H_{x} H_{a_{j}}=H_{x}$; hence $x a_{j} \in H_{x}$. If $x a_{j} \neq x$, then there is a semicharacter $\chi$ of $G^{\prime}$ such that $\chi(x) \chi\left(a_{j}\right)=\chi\left(x a_{j}\right) \neq \chi(x)$, by 5.6. Since $x a_{j} \in H_{x}$, we have $\chi\left(x a_{j}\right) \neq 0$, $\chi(x) \neq 0$. Therefore $\chi\left(a_{j}\right)=1$, since $a_{j}^{2}=a_{j}$, and $\chi(x) \neq \chi(x)$. From this contradiction, we infer that $x a_{j}=x$ and that $\left\{a_{1}, a_{2}, \cdots, a_{n}\right\}$ is a finite set of relative units for $G^{\prime}$. Then apply 7.5 .

8.14. Theorem. Let $G$ be a commutative semigroup. Then $\widehat{G}$ is compact if and only if there exists a finite subset $A=\left\{a_{1}, a_{2}, \cdots, a_{n}\right\}$ of $G$ such that for all $x \in G$, there exists $a_{j} \in A$ for which $x \in T_{a_{j} x}$.

Proof. This follows at once from 8.12, 8.7, and 4.4. 
8.15. TheOREM. Let $G$ be a commutative semigroup. Then $\widehat{G}$ is a compact semigroup if and only if $G^{\prime}$ has a unit.

Proof. In view of 8.6 , we may restrict our attention to $G^{\prime}$ and $\widehat{G}^{\prime}$. If $G^{\prime}$ has a unit, then it is obvious that $\widehat{G^{\prime}}$ is a semigroup. Also $l_{1}\left(G^{\prime}\right)$ in this case has a unit, and $\widehat{G^{\prime}}$ is compact. Conversely, suppose that $\widehat{G}^{\prime}$ is compact and a semigroup. Since $\widehat{G^{\prime}}$ is a semigroup, it follows from 6.7 that there is at most one maximal element in $G^{\prime \prime}$. Since $\widehat{G^{\prime}}$ is compact, there is at least one maximal element in $G^{\prime \prime}$. Let $H_{a}$, then, be the unique maximal element in $G^{\prime \prime}$. Since $\widehat{G^{\prime}}$ is compact, it follows as in the proof of 8.12 that $H_{a}$ is a group; we take $a$ to be the unit of $H_{a}$. The argument of 8.13 may be applied to show that $a$ is the unit of $G^{\prime}$. This completes the proof.

8.15.1. Remark. Semigroup 17 of Appendix 2 in [3] shows that $\widehat{G}$ may be compact while $l_{1}(G)$ has no unit. Hence we cannot replace $G^{\prime}$ by $G$ in 8.15 .

8.16. Theorem. Let $G$ be a commutative semigroup. Then $\widehat{G}$ is a compact semigroup if and only if there exists an element $a \in G$ such that for all $x \in G$, $a x \in T_{x}$.

Proof. Apply 8.15 and 4.4 .

9. Idempotents in $l_{1}\left(G^{\prime}\right)$. We discuss in this section the existence of idempotents in $l_{1}\left(G^{\prime}\right)$. As usual, a proper idempotent element $E$ of an algebra is taken to be an element different from the unit (if it exists) and from 0 such that $E^{2}=E$. Our main theorem is the following.

9.1. THEOREM. Let $G$ be a commutative semigroup such that $T_{x}=\{x\}$ for all $x \in G$. Then $l_{1}(G)$ contains a proper idempotent if and only if $G$ contains an element a of finite order that is not a unit for $G$.

It is convenient to break the proof up into several theorems.

9.2. THEOREM. The condition given in 9.1 is sufficient, even without the hypothesis $T_{x}=\{x\}$.

Proof. Let $a$ be an element of $G$ having finite order. If $a$ is idempotent and is not a unit for $G$, then $\boldsymbol{\lambda}_{a}$ is idempotent in $l_{1}(G)$ and, since $\boldsymbol{\lambda}_{a} * \boldsymbol{\lambda}_{x}=\boldsymbol{\lambda}_{a x} \neq \boldsymbol{\lambda}_{x}$ for some $x \in G, \lambda_{a}$ is a proper idempotent. If $a$ is not an idempotent of $G$, let $k$ and $l$ be the smallest positive integers such that $a^{k+l}=a^{l}$. If $k=1$, then $a^{l}$ is an idempotent of $G$ that is not a unit for $G$. If $k>1$, the set $\left\{a^{l}, a^{l+1}, \cdots\right.$, $\left.a^{l+k-1}\right\}=B$ is a finite subgroup of $G$ of order $>1$, and $E=(1 / k) \sum_{x \in B} \lambda_{x}$ is a proper idempotent of $l_{1}(G)$. (For the properties of $k$ and $l$ used here, see $[3,2.6]$.)

9.3. To show that the condition given in 9.1 is necessary, we first prove a preliminary fact. If $G$ is as in 9.1 , and if $z \in G$, we let $V_{z}$ denote the union of all of the sets $H_{x}$ for which $H_{z} H_{x}=H_{z}$.

9.4. TheOREM. Let $G$ be as in 9.1 , and let $E=\sum_{n-1}^{\infty} \epsilon_{n} \lambda_{x_{n}}$ be an idempotent 
element of $l_{1}(G)$. For an arbitrary $z \in G$, let $A=\sum_{n}^{(z)} \epsilon_{n} \lambda_{x_{n}}$, the symbol $\sum_{n}^{(z)} d e-$ noting the sum over all $n$ such that $x_{n} \in V_{\mathbf{z}}$. Then $A$ is also an idempotent element of $l_{1}(G)$.

Proof. It is not hard to show that $V_{2}$ is a subsemigroup of $G$ whose complement is an ideal. If $\psi_{z}$ is a semicharacter of $V_{z}$, then the function $\psi$ such that $\psi(x)=\psi_{z}(x)$ for $x \in V_{z}$ and $\psi(x)=0$ otherwise is a semicharacter of $G$ (6.4). We further have $\widehat{E}(\psi)=\widehat{A}\left(\psi_{z}\right)$, when we regard $A$ as an element of $l_{1}\left(V_{z}\right)$. Since $E$ is idempotent, the last equality implies that $\widehat{A}\left(\psi_{z}\right)$ assumes only the values 0 and 1 . Therefore $\widehat{A}^{2}=\widehat{A}$, and since $l_{1}\left(V_{z}\right)$ is semisimple (5.6.2), it follows that $A$ is idempotent.

9.5. THEOREM. Let $G$ be as in 9.1 , let $G$ contain a unit e, and no other element of finite order. Then the complement $P$ of $H_{e}$ in $G$ is a subsemigroup. If $l_{1}(G)$ contains a proper idempotent $E=\sum_{n=1}^{\infty} \epsilon_{n} \lambda_{x_{n}}$, then $l_{1}(P)$ contains a proper idempotent.

Proof. It is clear that $H_{e}=V_{e}$, and, as noted in the proof of $9.4, P$ is even an ideal in $G$. Let $A$ be defined with respect to $z=e$ as in 9.4. Then $A$ is an idempotent in $l_{1}\left(H_{e}\right)$. If $A$ were a proper idempotent, then $\widehat{A}$ would assume both the values 0 and 1 , and $\widehat{H}_{e}$ would be disconnected. However, by hypothesis $H_{e}$ contains no element of finite order other than $e$, and $H_{e}$ is a group (4.16). These conditions imply that $\widehat{H}_{e}$ is connected $[9, \mathrm{p} .110]$. It follows that $A=0$ or $A=\lambda_{e}$. If $A=0$, then $E$ may be regarded as a nonzero element of $l_{1}(P)$. As $P$ contains no idempotent elements, $l_{1}(P)$ has no unit (7.3), and $E$ is a proper idempotent of $l_{1}(P)$. If $A=\lambda_{e}$, then $\lambda_{e}-E$ is plainly idempotent and different from 0 . This functional too may be regarded as an element of $l_{1}(P)$. As in the case $A=0, \lambda_{e}-E$ must be a proper idempotent of $l_{1}(P)$.

9.6. ThEOREM. Let $G$ be as in 9.1, and let $G$ contain no element of finite order. Then $l_{1}(G)$ contains no proper idempotent.

Proof. The proof is by contradiction. Assume that $E \in l_{1}(G)$, that $E^{2}=E$, and that $E \neq 0$. We write $E$ in the usual way as $\sum_{n} \epsilon_{n} \lambda_{x_{n}}$, the sum being over just those positive integers $n$ for which $\epsilon_{n}$ is different from 0 . There exists a positive integer $m$ such that $\sum_{n>m}\left|\epsilon_{n}\right|<1 / 2$ (a void sum being 0 ). It is not hard to show that there exists an $x_{j}(1 \leqq j \leqq m)$ having the property that the equality $H_{x_{j}} H_{x_{n}}=H_{x_{j}}$ implies that $H_{x_{n}}=H_{x_{j}}$ or that $n$ is greater than $m$. We omit the details of this argument. We write the $x_{j}$ so selected as $z$ and construct $A \in l_{1}(G)$ from $E$ and $z$ as in 9.4. Thus

9.6 .1

$$
A=\sum_{n}^{(z)} \epsilon_{n} \lambda_{x_{n}} \neq 0 .
$$

Let $C=\sum_{x_{n} \in H z} \epsilon_{n} \lambda_{x_{n}}$ and let $D=A-C$. Then $D=\sum_{n} \epsilon_{n} \lambda_{x_{n}}$, where the sum is taken over all $n$ such that $x_{n} \notin H_{z}$ and $x_{n} \in V_{z}$. Our construction implies 
that all of these $n$ 's are greater than $m$. For an arbitrary semicharacter $\chi$ of $G$, we clearly have

$$
\widehat{A}(\chi)=\widehat{C}(\chi)+\widehat{D}(\chi), \text { and }|\widehat{D}(\chi)|<1 / 2 .
$$

There are now two cases to consider. First, if $\chi(z)=0$, we know from 5.1.1 that $\chi\left(x_{n}\right)=0$ for all $x_{n} \in H_{z}$. Hence $\widehat{C}(\chi)=0$, and $|\widehat{A}(\chi)|<1 / 2$ by 9.6.2. Since $A$ is idempotent, we have $\widehat{A}(\chi)=0$ or 1 , and therefore $\widehat{A}(\chi)=0$.

The second case, $\chi(z) \neq 0$, requires a more elaborate argument. Let $\chi_{0}$ be the semicharacter of $H_{z}$ such that $\chi_{0}(x)=\chi(x)$ for all $x \in H_{z}$. Since $H_{z}$ contains no idempotent, it is a non-group, and by 8.10 and the fact that the absolute value of a semicharacter is again a semicharacter, we see that there exists a semicharacter $\chi_{1}$ of $H_{z}$ such that $0<\chi_{1}(x)<1$ for all $x \in H_{z}$. Then, for $0 \leqq r<\infty$, the function $\chi^{(r)}$, defined by the relations

$$
\chi^{(r)}(x)=\left\{\begin{array}{l}
0 \text { if } H_{z} H_{x} \neq H_{z}, \\
\frac{\chi_{0} \chi_{1}^{r}(z x)}{\chi_{0} \chi_{1}^{r}(z)} \text { if } H_{z} H_{x}=H_{z},
\end{array}\right.
$$

is a semicharacter of $G(6.5)$. Then we have

$$
\begin{aligned}
\widehat{A}(\chi) & =\sum_{n}^{(z)} \epsilon_{n} \chi\left(x_{n}\right)=\sum_{n}^{(z)} \epsilon_{n} \frac{\chi\left(z x_{n}\right)}{\chi(z)}=\sum_{n}(z) \epsilon_{n} \frac{\chi_{0}\left(z x_{n}\right)}{\chi_{0}(z)} \\
& =\sum_{n} \epsilon_{n} \chi^{(0)}\left(x_{n}\right)=\widehat{A}\left(\chi^{(0)}\right) .
\end{aligned}
$$

Now the function $\widehat{A}\left(\chi^{(r)}\right)$, given by

$$
\widehat{A}\left(\chi^{(r)}\right)=\sum_{n}^{(z)} \epsilon_{n} \frac{\chi_{0}\left(z x_{n}\right) \chi_{1}^{r}\left(z x_{n}\right)}{\chi_{0}(z) \chi_{1}^{r}(z)},
$$

is a continuous function of $r$ for $r \geqq 0$, and hence we have $\widehat{A}\left(\chi^{(r)}\right)=0$ for all $r \geqq 0$ or $\widehat{A}\left(\chi^{(r)}\right)=1$ for all $r \geqq 0$. Furthermore, we have $\left|\widehat{C}\left(\chi^{(r)}\right)\right|$ $=\left|\sum_{x_{n} \in H_{z}} \epsilon_{n} \chi_{0}\left(x_{n}\right) \chi_{1}^{r}\left(x_{n}\right)\right|<1 / 2$ for all $r$ sufficiently large. By 9.6 .2 with $\chi=\chi^{(r)}$, we have $\left|\widehat{A}\left(\chi^{(r)}\right)\right|<1$ for $r$ sufficiently large, and hence $\widehat{A}\left(\chi^{(r)}\right)=0$ for all $r \geqq 0$. From 9.6.3, it follows that $\widehat{A}(\chi)=0$.

Putting these 2 cases together, we find that $\widehat{A}=0$, and since $l_{1}(G)$ is semisimple, we have $A=0$. This contradicts 9.6 .1 , and therefore the assumption $E \neq 0$ is false.

9.7. Proof of 9.1. The sufficiency argument is given in 9.2. To establish the necessity of the condition given in 9.1 , let $E$ be a proper idempotent of $l_{1}(G)$. If the only element of finite order in $G$ is the unit of $G$, then 9.5 reduces our problem to the case treated in 9.6.

10. Connectedness of $\widehat{G}_{0}$.

10.1. Silov has shown $[7$, p. 362] that if $A$ is a commutative Banach algebra with unit, and if the maximal ideal space of $A$ is disconnected, then 
$A$ contains a proper idempotent. The converse, which is very simple, was noted by Gel'fand [2, p. 19]. Silov's theorem is not true for commutative Banach algebras without unit, but something very like it is true. We discuss this matter here and apply the result, together with 9.1, to finding conditions under which $\widehat{G}$ is disconnected.

10.2. TheOREM. Let $A$ be a semisimple commutative Banach algebra without unit. If $A$ contains an idempotent e different from 0 , then the maximal ideal space $\mathfrak{M}$ of $A$ is disconnected. More specifically, there exist nonvoid complementary closed subsets of $\mathfrak{M}$, one of which is compact.

We omit the proof.

10.3. Remark. Theorem 10.2 obviously fails if $A$ is not semisimple.

10.4. ThEOREM. Let $A$ be a commutative Banach algebra with or without unit, and suppose that the maximal ideal space $\mathfrak{M}$ of $A$ is disconnected, $\mathfrak{M}=\mathfrak{F}_{1} \cup \mathfrak{F}_{2}$, where $\mathfrak{F}_{1}$ and $\mathfrak{F}_{2}$ are nonvoid, closed, and complementary. If at least one of $\mathfrak{F}_{1}$ and $\mathfrak{F}_{2}$ is compact, then $A$ contains an idempotent $f$ that is different from 0 and from the unit of $A$ if one exists.

Proof. If $A$ has a unit, then we have only to cite Silov's theorem as in 10.1. In this case $\mathfrak{M}$ and hence $\mathfrak{F}_{1}$ and $\mathfrak{F}_{2}$ are compact. If $A$ has no unit, we adjoin a unit $e$, obtaining the algebra $A_{\text {o }}$ of all elements $x+\lambda e$, where $x \in A$ and $\lambda$ is complex. The maximal ideal space of $A_{e}$ is $\mathfrak{M} \cup\{p\}$, where $p$ is an adjoined point representing the homomorphism $x+\lambda e \rightarrow \lambda$ of $A_{e}$ onto the complex number field. If $\mathfrak{F}_{1}$, say, is compact, it is clear that $\mathfrak{F}_{1}$ and $\mathfrak{F}_{2} \cup\{p\}$ are nonvoid complementary closed subsets of $\mathfrak{M} \cup\{p\}$. Applying Silov's theorem to $A_{e}$, we find that $A_{e}$ contains an idempotent $x+\lambda e$, where $x \neq 0$. Since $x+\lambda e=x^{2}+2 \lambda x+\lambda^{2} e$, we have $\lambda$ equal to 0 or 1 . If $\lambda=1$, then $-x$ is an idempotent in $A$. If $\lambda=0$, then $x$ is an idempotent in $A$. In either case, $A$ contains an idempotent $f \neq 0$, and since in the present case $A$ has no unit, the proof is complete.

10.5. REMARK. Theorem 10.4 fails to be true if both $\mathfrak{F}_{1}$ and $\mathfrak{F}_{2}$ are noncompact. A simple example to show this is provided by the Banach algebra of all continuous complex-valued functions $f$ on $[0,1]$ such that $f(1 / 2)=0$, with pointwise operations and $\|f\|=\max _{0 \leqq t \leqq 1}|f(t)|$. The maximal ideal space here is the space $[0,(1 / 2)[U](1 / 2), 1]$, with the usual topology, and is obviously disconnected. However the algebra in question contains no idempotent elements except 0 .

The foregoing results may be applied to $\widehat{G}$ as follows.

10.6. THEOREM. Let $G$ be a commutative semigroup. Then $\widehat{G}$ is the union of 2 nonvoid complementary closed sets, one of which at least is compact, if and only if $G^{\prime}$ contains an element of finite order that is not a unit for $G^{\prime}$.

Proof. This assertion follows directly from 10.2, 10.4, and 9.1. 
11. Examples. We now take up various examples that illustrate the foregoing theory. We begin with the simple case of a commutative idempotent semigroup.

11.1. According to $[3,2.7]$, every commutative idempotent semigroup $G$ admits a faithful representation by a semigroup $\{A\}$ consisting of sets, the semigroup operation in $\{A\}$ being set-theoretic intersection.

11.1.1. It is not hard to see that the semirharacters of $\{A\}$ correspond in a 1-to-1 way with filters in $\{A\}$. A filter in $\{A\}$ is a nonvoid subfamily $\{B\}$ of $\{A\}$ such that: (1) $B_{1}, B_{2} \in\{B\}$ imply $B_{1} \cap B_{2} \in\{B\}$; (2) $B \in\{B\}$, $C \supset B$, and $C \in\{A\}$ imply $C \in\{B\}$. Every such filter is the set where a semicharacter assumes the value 1 , and conversely.

11.1.2. The representation $u \rightarrow \widehat{\lambda_{u}}$ of an idempotent commutative semigroup $G$ is faithful, since $l_{1}(G)$ is semisimple. Since $\widehat{\lambda}_{u}$ assumes only the values 0 and 1 , the representation $u \rightarrow\left\{\chi ; \chi \in \widehat{G}, \widehat{\lambda}_{u}(\chi)=1\right\}$ is a faithful representation of $G$ by subsets $A$ of $\widehat{G}$. In this representation, all filters and hence all semicharacters are described by families of sets of the form $\left\{A ; \chi_{0} \in A\right\}$ for some fixed $\chi_{0} \in \widehat{G}$. This corresponds to Stone's perfect representation of a Boolean algebra. (See [8, p. 107], and also [3, 2.7.2].)

11.1.3. The semigroup of all real numbers $t$, with the semigroup operation $s \cdot t=\max (s, t)$ is an example of a semigroup in which the equation $a y=a$ has an infinite number of solutions for all $a$ in the semigroup. By using wellordered sets, with the operation $\gamma \cdot \delta=\max (\gamma, \delta)$, one easily gets semigroups in which this equation has a set of solutions of arbitrarily large cardinal number.

11.1.4. Let $G$ be the positive integers $\{1,2,3, \ldots\}$ with the semigroup operation $\max (x, y)$. This semigroup has the unit 1 . It is simple to show that the functions $\chi_{k},(k=1,2,3, \cdots)$, where $\chi_{k}(x)=1$ for $1 \leqq x \leqq k$ and $\chi_{k}(x)=0$ for $x>k$, are semicharacters. The only other semicharacter is the function 1 . The topology of $\widehat{G}$ makes every $\chi_{k}$ isolated, and a generic neighborhood of 1 is $\left\{\chi_{s}, \chi_{s+1}, \cdots\right\}$ for a positive integer $s$. Thus $\widehat{G}$ is compact. This is in consonance with 8.7. For $A=\sum_{n=1}^{\infty} \alpha_{n} \lambda_{n} \in l_{1}(G)$, we have $\widehat{A}(1)$ $=\sum_{n=1}^{\infty} \alpha_{n}$, and $\widehat{A}\left(\chi_{k}\right)=\sum_{n=1}^{k} \alpha_{n}$. The class of Fourier transforms in this case may be represented as the class of all functions $f$ defined on $\{1,2,3, \ldots, \infty\}$ such that $\lim _{n \rightarrow \infty} f(n)=f(\infty)$ and $\sum_{n=1}^{\infty}|f(n+1)-f(n)|$ is finite. If $\sum_{n=1}^{\infty} u_{n}$ is a conditionally convergent infinite series and $g(k) \supseteq \sum_{n=1}^{k} u_{n}$ for $k=1,2,3, \cdots, g(\infty)=\sum_{n=1}^{\infty} u_{n}$, then $g$ is a continuous function on $\widehat{G}$ that is not a Fourier transform.

11.1.5. Let $G$ be the positive integers $\{1,2,3, \ldots\}$ with the semigroup operation $\min (x, y)$. This semigroup has no finite set of relative units. Accordingly, by $7.3, l_{1}(G)$ has no unit, and by $8.12, \widehat{G}$ is not compact. In this case it is easy to show that the functions $\psi_{n}$, where $\psi_{n}(x)=0$ for $1 \leqq x \leqq n$ and $\psi_{n}(x)=1$ for $x>n$, are semicharacters and that these are the only semicharacters. The space $\widehat{G}$ is discrete and hence locally compact. For $A=\sum_{n=1}^{\infty} \alpha_{n} \lambda_{n}$ $\in l_{1}(G)$, we have $\widehat{A}\left(\psi_{k}\right)=\sum_{n=k}^{\infty} \alpha_{k}$. 
11.1.6. Let $G$ be any nonvoid set and let $a$ be a fixed element of $G$ : let $x^{2}=x$ for all $x \in G$ and $x y=a$ if $x \neq y$. Then $G$ is a commutative idempotent semigroup. The function 1 is a semicharacter of $G$; every other semicharacter of $G$ has the form $\chi_{y}$ for some $y \neq a$, where $\chi_{y}(x)=\delta_{x y}$ for all $x \in G$. If $G$ is infinite, then $G$ has no finite set of relative units, $l_{1}(G)$ has no unit, $\widehat{G}$ has the discrete topology, and of course $l_{1}(G)$ contains a large number of proper idempotents. If $G$ is finite, $G=\left\{a, x_{1}, x_{2}, \cdots, x_{p}\right\}$, then the unit of $l_{1}(G)$ is $E=\lambda_{x_{1}}+\cdots+\lambda_{x_{p}}-(p-1) \lambda_{a}$, and $\|E\|=2 p-1$.

11.2. By slightly altering the definition of multiplication given in 11.1.6, we obtain a very different $G$ and $l_{1}(G)$. For $G$ any nonvoid set, let $a$ be a fixed element of $G$ and let $x y=a$ for all $x, y \in G$. The function 1 is the only semicharacter of $G$, and $G^{\prime}, G^{\prime \prime}$, and $G^{0}$ all consist of just one element. For all $A=\sum_{x \in G} \alpha(x) \lambda_{x} \in l_{1}(G)$ and $u \in G$, we have $\lambda_{u} * A=\left(\sum_{x \in G} \alpha(x)\right) \lambda_{a}$, so that $l_{1}(G)$ has no unit if $G$ contains more than one element. The semigroup $\widehat{G}$ consists of exactly one element.

11.3. If $G$ is a commutative semigroup in which $T_{x}=\{x\}$, then there are an infinite number of ways to imbed $G$ in a semigroup that is a union of groups. For example, one may adjoin in succession as many zeros as one wishes to the semigroup $G^{0}[3,2.2]$. A simple nontrivial example of 2 distinct imbeddings is provided by the additive semigroup $\{0,1,2,3, \ldots\}$. Obviously this semigroup can be imbedded in the additive group $\{\cdots,-2,-1$, $0,1,2, \cdots\}$. However we have $H_{0}=\{0\}$ and $H_{1}=\{1,2,3, \cdots\}$, and $G^{0}$ is the union of $H_{0}^{0} \cong\{0\}$ and $H_{1}^{0} \cong\{\cdots,-2,-1,0,1,2, \cdots\}$.

11.4. The semigroup $G=\{1,2,3, \ldots\}$ under addition is clearly a semigroup in which $T_{x}=\{x\}$ and $H_{1}=H_{2}=\cdots=G$. A semicharacter $\chi_{z}$ of $G$ is determined by the complex number $z, 0<|z| \leqq 1$, such that $\chi_{z}(1)=z$. The semigroup $\widehat{G}$ is thus isomorphic to the multiplicative semigroup $\{z ; z$ is complex, $0<|z| \leqq 1\}$. The topology of $\widehat{G}$ is easily seen to be that of $\widehat{G}$ as a subset of the plane. The algebra $l_{1}(G)$ is of course very well known (see for example $\left[10\right.$, p. 444]); it is isomorphic to the algebra of all functions $f(z)=\sum_{n=1}^{\infty} \alpha_{n} z^{n}$ such that $\sum_{n=1}^{\infty}\left|\alpha_{n}\right|$ is finite. This semigroup is the simplest example of a commutative semigroup that is a nongroup and that is a single $H_{x}$. Since $G^{\prime \prime}$ consists of a single element, $l_{1}\left(G^{\prime \prime}\right)$ has a unit, but $\widehat{G}$ is not compact. Previously established facts are all apparent here: semicharacters exist that everywhere have absolute value less than 1 ; no semicharacter vanishes anywhere; the cancellation law holds; no element is idempotent. Note too that $l_{1}(G)$ contains no idempotent except 0 , and that $\widehat{G}$ is connected.

11.5. The additive semigroup $G=\{0,1,2,3, \ldots\}$ is very like $\{1,2,3, \cdots\}$, being obtained from $\{1,2,3, \cdots\}$ by the adjunction of the unit 0 . Here we have $\widehat{G} \cong D(8.3)$, with the usual topology, the function $\chi_{0}$ such that $\chi_{0}(0)=1, \chi_{0}(k)=0$ for $k=1,2,3, \cdots$ being a semicharacter of $G$. As remarked in $11.3, G^{0}$ is a nongroup. The algebra $l_{1}(G)$ has a unit, $\lambda_{0}$, 
has no proper idempotents, and is isomorphic to the algebra of all functions $f(z)=\sum_{n=0}^{\infty} \alpha_{n} z^{n}, \sum_{n=0}^{\infty}\left|\alpha_{n}\right|<\infty$.

11.6. A complete classification of all commutative semigroups $G$ in which $T_{x}=\{x\}$ and $G$ is a single $H_{x}$ would be very complicated. These semigroups come very close, in a sense, to being groups, as 4.16, 5.1.1, and 8.10 show. Algebraically, the relationship with groups may be rendered precise as follows. A commutative semigroup is a group if and only if the equation $a u=b$ has a solution $u$ for all $a$ and $b$. A commutative semigroup in which $T_{x}=\{x\}$ is a single $H_{x}$ if and only if the 2 equations $a^{n}=u b, b^{n}=v a$ have solutions $u, v$, and $n$ for all $a$ and $b$.

11.6.1. Non-groups of the kind under study are easy to find, as noted in 11.4 , and may have arbitrarily large cardinal number. For example, let $X$ be any nonvoid set, and let $G$ be the additive semigroup of all bounded realvalued functions $f$ on $X$ for each of which there exists a positive number $\delta$ such that $f(x) \geqq \delta$ for all $x \in G$.

11.6.2. The example $\{0,1,2,3, \cdots\} \subset\{\cdots,-2,-1,0,1,2, \cdots\}$ shows that a subsemigroup of a semigroup having the property under study may fail to have it.

11.7. Let $G$ be an arbitrary commutative semigroup and let $G \cup\{a\}$ be obtained from $G$ by adjoining a repeat element, as in 2.4 of [3]. Then we have $a \in T_{x_{1}}$, so that $l_{1}(G \cup\{a\})$ is never semisimple, by 5.9.

\section{REFERENCES}

1. A. H. Clifford, Semigroups admitting relative inverses, Ann. of Math. Ser. 2, vol. 2 (1941) pp. 1037-1049. 3-24.

2. I. M. Gel'fand, Normierte Ringe, Rec. Math. (Mat. Sbornik) N. S. vol. 9 (1941) pp.

3. Edwin Hewitt and H.S. Zuckerman, Finite dimensional convolution algebras, Acta Math. vol. 93 (1955) pp. 67-119.

4. Einar Hille, Functional analysis and semi-groups, Amer. Math. Soc. Colloquium Publications, no. 31, New York, 1948. 1953.

5. Lynn H. Loomis, An introduction to abstract harmonic analysis, New York, van Nostrand,

6. Wilhelm Maak, Fastperiodische Funktionen, Die Grundlehren der Mathematischen Wissenschaften in Einzeldarstellungen, vol. 61, Berlin-Göttingen-Heidelberg, Springer-Verlag, 1950.

7. G. E. Šilov, O razloženii kommutativnogo normirovannogo kol'ca v pryamuyu summu idealov, Rec. Math. (Mat. Sbornik) N. S. vol. 32 (1953) pp. 353-364.

8. M. H. Stone, The theory of representations for Boolean algebras, Trans. Amer. Math. Soc. vol. 40 (1936) pp. 37-111.

9. André Weil, L'intégration dans les groupes topologiques et ses applications, Actualités Scientifiques et Industrielles no. 869, Paris, Hermann, 1940.

10. D. V. Widder, The convolution transform, Bull. Amer. Math. Soc. vol. 60 (1954) pp. 444-456.

UNIVERSITY OF WASHINGTON,

Seattle, Wash. 